\title{
1 Exploring the interaction network of a synthetic gut bacterial
}

\section{2 community}

4 Anna S. Weiss ${ }^{1}$, Anna G. Burrichter ${ }^{1^{*}}$, Abilash Chakravarthy Durai Raj ${ }^{{ }^{*}}$, Alexandra von

5 Strempel ${ }^{1}$, Chen Meng ${ }^{2}$, Karin Kleigrewe ${ }^{2}$, Philipp C. Münch ${ }^{1,3}$, Luis Rössler ${ }^{4}$, Claudia

6 Huber ${ }^{4}$, Wolfgang Eisenreich ${ }^{4}$, Lara M. Jochum ${ }^{5}$, Stephanie Göing ${ }^{6}$, Kirsten Jung ${ }^{6}$, Alvaro

$7 \quad$ Sanchez ${ }^{7,8}$, Bärbel Stecher ${ }^{1,9}$

$9 \quad$ * These authors contributed equally to this work.

$11{ }^{1}$ Max von Pettenkofer Institute of Hygiene and Medical Microbiology, Faculty of Medicine,

12 LMU Munich, Germany

$13 \quad{ }^{2}$ Bavarian Center for Biomolecular Mass Spectrometry, TU Munich, Freising, Germany

$14{ }^{3}$ Department for Computational Biology of Infection Research, Helmholtz Center for

15 Infection Research, Brunswick, Germany

$16{ }^{4}$ Department of Chemistry, Bavarian NMR Center - Structural Membrane Biochemistry, TU

17 Munich, Garching, Germany

$18 \quad{ }^{5}$ Ramboll Deutschland GmbH, Munich, Germany

$19{ }^{6}$ Department of Microbiology, LMU, Martinsried, Germany

$20{ }^{7}$ Department of Ecology \& Evolutionary Biology, Yale University, New Haven, CT, USA

$21 \quad{ }^{8}$ Microbial Sciences Institute, Yale University, West Haven, CT, USA

$22{ }^{9}$ German Center for Infection Research (DZIF), partner site LMU Munich, Germany 
Metabolic network, minimal consortium, syncom, cross-feeding, microbial community

A key challenge in microbiome research is to predict functionality from microbial community composition. As central microbiota functions are determined by bacterial community networks it is important to gain insight into the principles that govern bacteria-bacteria interactions. Here, we focused on growth and metabolic interactions of the Oligo-Mouse-Microbiota (OMM ${ }^{12}$ ) synthetic bacterial community, which is increasingly used as model system in gut microbiome research. Using a bottom-up approach, we uncovered the directionality of strain-strain interactions in mono- and pairwise co-culture experiments, as well as in community batch culture. Metabolomics analysis of spent culture supernatant of individual strains in combination with genome-informed pathway reconstruction provided insights into the metabolic potential of the individual community members. Thereby, we could show that the $\mathrm{OMM}^{12}$ interaction network is shaped by both, exploitative and interference competition in vitro. In particular, Enterococcus faecalis KB1 was identified as important driver of community composition by affecting the abundance of several other consortium members. Together, this study gives fundamental insight into key drivers and mechanistic basis of the $\mathrm{OMM}^{12}$ interaction network, which serves as knowledge base for future mechanistic studies.

\section{Introduction}

The mammalian gastrointestinal tract harbors hundreds of bacterial species that occupy distinct ecological niches $(1,2)$. Diversity and stable coexistence of community members after initial assembly result in exclusion of invaders $(3,4)$. Community assembly and stability are inherently driven by commensal or cooperative trophic interactions, in which metabolic by- or end products of one species are the resource for another one (5-7). At the same time, bacteria compete for substrates by employing diverse predatory mechanisms, like the production of bacteriocins (8). These interaction patterns form complex ecological networks and determine community-level functions of the microbiota including dietary breakdown, metabolite production and colonization resistance (9-11). Consequently, disruption of bacterial networks by antibiotics, disease or diet-mediated interventions results in impairment of community-level functions $(12,13)$. To be able to predict, preserve and manipulate microbial community function, it is important to identify functionally important members and understand relevant interaction mechanisms between individual bacteria.

A multitude of different approaches have been used to characterize ecological networks of microbial communities. Function-related patterns in native microbial communities can been identified by systems biology approaches, combining metagenomics, metatranscriptomics and metabolomics analyses (14). Together with stable-isotope probing methodologies microorganisms with specific metabolic properties can be identified (15). Potentially interacting species may be predicted from co-occurrence analysis supported by genome guided 
metabolic modeling (16-18). To experimentally verify the key ecological, structural and functional role of certain species in community structure and function, synthetic microbial consortia provide several advantages over native communities. As they are well-characterized, scalable and experimentally tractable, these systems are increasingly used to gain a mechanistic understanding of gut microbial ecology (19-22).

The Oligo-Mouse-Microbiota $\left(\mathrm{OMM}^{12}\right)$ is a synthetic bacterial community, which stably colonizes mice and provides colonization resistance against enteropathogen infection (23-26). The $\mathrm{OMM}^{12}$ comprises twelve bacterial species (Enterococcus faecalis $\mathrm{KB} 1$, Limosilactobacillus reuteri I49, Bifidobacterium animalis YL2, Clostridium innocuum I46, Blautia coccoides YL58, Enterocloster clostridioformis YL32, Flavonifractor plautii YL31, Acutalibacter muris KB18, Bacteroides caecimuris I48, Muribaculum intestinale YL27, Akkermansia muciniphila YL44 and Turicimonas muris YL45), representing the five major eubacterial phyla in the murine gastrointestinal tract (27) (Fig. 1A). The model is freely available for non-commercial use (28), and is therefore increasingly employed in preclinical microbiome research (29-32). So far little is known about the system's ecology and metabolic capabilities, both of which are factors that determine assembly, population dynamics and bacterial community functionality. Therefore, we aimed for a comprehensive exploration of the metabolic potential (i.e., substrates, metabolism and end products) and interactions between individual members of the $\mathrm{OMM}^{12}$ consortium. We employed a bottom-up approach connecting outcomes of mono- and pairwise co-culture experiments with observations from complex communities in in vitro batch culture. Furthermore, we combined metabolomics analysis of spent culture supernatants with genome-informed pathway reconstruction and generated draft metabolic models of the $\mathrm{OMM}^{12}$ consortium. Overall, we find that the majority of in vitro strain-strain interactions is amensalistic or competitive. In accordance, bacteriocin production and substrate overlap between the individual strains was correlated with negative strain-strain interaction in vitro, revealing potentially underlying mechanisms. Together, this work identified key interaction patterns among $\mathrm{OMM}^{12}$ strains relevant in community assembly and functionality.

\section{Results}

\section{Probing directional interactions of $\mathrm{OMM}^{12}$ strains using spent culture media}

To characterize directional interactions of the $\mathrm{OMM}^{12}$ consortium members, we chose an in vitro approach to explore how the bacterial strains alter their chemical environment by growth to late stationary phase.

Growth of the individual monocultures in a rich culture medium (AF medium, Methods, Tab. S1, Tab. S2) was monitored over time (Fig. S1) and growth rates (Tab. S3) were determined. Strains were grouped by growth rate (GR) into fast growing strains (GR $>1.5 \mathrm{~h}^{-1}$, E. faecalis KB1, B. animalis YL2, C. innocuum I46 and B. coccoides YL58), strains with intermediate growth rate (GR $>1 \mathrm{~h}^{-1}, M$. intestinale YL27, $F$. plautii YL31, E. clostridioformis YL32, B. caecimuris I48 and L.reuteri I49) and slow growing strains (GR $<1 \mathrm{~h}^{-1}, A$. muris KB18, A. muciniphila YL44 and T. muris YL45). All strains reached late stationary phase within $20 \mathrm{~h}$ of growth. To probe overlap in substrate requirements and interactions between the individual $\mathrm{OMM}^{12}$ members mediated by waste products or bacteriocins, sterile spent culture medium (SM) after growth to late stationary phase of all strains was obtained. Each $\mathrm{OMM}^{12}$ strain was 
109 cultured in the SM of the other community members and their own SM and growth rate, the area under the growth curve (AUC) and the $\mathrm{pH}$ were determined (Fig. 1B, Fig. S2).

111 A normalized inhibition factor ( $\mathrm{d}_{\mathrm{AUC}}$ ) was determined by the AUC in SM relative to the AUC 112 in fresh AF medium ( $\left.\mathrm{d}_{\mathrm{AUC}}=\frac{A U C_{S M}-A U C_{A F}}{A U C_{A F}}\right)$ to quantify the influence of the different SM on the

113 growth of the individual $\mathrm{OMM}^{12}$ strains (Fig. 1C). Ten of the twelve SM were found to strongly decrease $\left(d_{\text {AUC }}<-0.5\right)$ the growth of at least one other strain of the consortium. Only the SM of strains A. muris KB18 and A. muciniphila YL44 were found to strongly inhibit growth of just the strains themselves. Corresponding to decreased AUC values in SM, growth rates were found to be lower as well, resulting in linear correlation of AUC and growth rates (Fig. S3, R $>0.5, \mathrm{p}<0.05$ for all strains). The SM of four strains, E. faecalis KB1, B. coccoides YL58, E. clostridioformis YL32 and B. caecimuris I48, were found to strongly inhibit ( $\left.\mathrm{d}_{\mathrm{AUC}}<-0.5\right)$ the growth of nine other strains each (Fig. 1C). Notably, growth of E. faecalis KB1 itself was only strongly reduced in its own SM, while it was able to grow in other strains' SM. T. muris YL45 was the only strain not showing clear growth inhibition in any of the SM while its SM strongly decreased growth $\left(\mathrm{d}_{\mathrm{AUC}}<-0.5\right)$ of three other strains, A. muris KB18, M. intestinale YL27 and F. plautii YL31.

\section{Individual $\mathrm{pH}$ profiles as indicators for niche modification}

The $\mathrm{pH}$ of the culture medium after growth to stationary phase can be used as a measure for the extent of strain specific environmental modification (11) and may partly explain inhibition of bacterial growth in a SM. Therefore, we determined the $\mathrm{pH}$ of the individual SM before and after (double spent media; DSM) growth of all OMM ${ }^{12}$ strains (Fig. 1B, Methods). From these values, we defined the $\Delta \mathrm{pH}$ for every strain after growth in fresh medium $\left(\Delta \mathrm{pH}_{\mathrm{SM}}\right)$ and in all $\mathrm{SM}\left(\Delta \mathrm{pH}_{\mathrm{DSM}}\right)$ by analyzing the strength (difference of $\mathrm{pH}$ values) and direction (more acidic or more alkaline) of the $\mathrm{pH}$ change (Fig. 1D). After growth in fresh AF medium with neutral $\mathrm{pH}$ of 7.0, the $\mathrm{OMM}^{12}$ strains showed different degrees of $\Delta \mathrm{pH}_{\mathrm{sM}}$. While E. faecalis KB1, $B$. animalis YL2, M. intestinale YL27, B. caecimuris I48 and B. coccoides YL58 distinctly acidified the medium $\left(\mathrm{pH}_{\mathrm{SM}}<6.2\right)$, the growth of the other strains resulted in either slightly more alkaline or nearly neutral medium. Correlating inhibition of growth in a SM ( $\left.\mathrm{d}_{\mathrm{AUC}}\right)$ with the mean $\mathrm{pH}$ of the individual SM for each strain revealed that growth inhibition did not directly correlate with the $\mathrm{pH}$. Only strains $B$. animalis YL2, A. muciniphila YL44 and B. caecimuris I48 showed a significant negative correlation $(\mathrm{R}<-0.5, \mathrm{p}<0.05)$ between growth inhibition and $\mathrm{pH}$ (Fig. S4) with stronger inhibition in more acidic $\mathrm{pH}$ ranges.

Most interestingly, many strains did not show the same magnitude or direction of alteration in $\mathrm{pH}$ when grown in $\mathrm{SM}$ of another strain $\left(\Delta \mathrm{pH} \mathrm{DSM}_{\mathrm{DS}}\right)$ compared to growth in fresh culture medium $\left(\Delta \mathrm{pH}_{\mathrm{SM}}\right)$. This indicates an altered metabolic behavior of some strains in specific $\mathrm{SM}$ environments that differs from metabolic behavior in fresh AF medium (Fig. S5, Supplemental Text A).

\section{Production of antibacterial compounds by $E$. faecalis KB1}

Growth inhibition in SM (Fig. 1C) can further be explained by the production of antimicrobial compounds. To test the production of antimicrobial compounds by the $\mathrm{OMM}^{12}$ strains, we used a phenotyping approach and performed spot assays on agar plates (Fig. 1E). Inhibition zones were only seen in case of $E$. faecalis KB1, which produced one or several compounds active against B. animalis YL2, F. plautii YL31, E. clostridioformis YL32, C. innocuum I46 and L. reuteri $\mathrm{I} 49$. Genome analysis revealed that the strain encodes genes for the production of several bacteriocins (Supplemental Text B), including enterocin L50, an enterococcal leaderless bacteriocin with broad target range among Gram-positive bacteria (33). All other strain pairs did not show signs of growth inhibition by compound excretion under these 
conditions, despite the presence of genes for lanthibiotic production in the genome of $B$. coccoides YL58 (determined by antiSMASH) (34). Although expression of antimicrobial molecules may be induced by specific environmental triggers which are absent in the monoculture in vitro setting, we concluded that interference competition may only play a role in a subset of pair-wise interactions in AF medium involving E. faecalis KB1.

\section{Substrate depletion profiles correlate with growth inhibition in SM}

As $\mathrm{pH}$ and antimicrobial compounds only partly explained inhibition of growth in SM, we set out to gain more insights into the individual metabolic profiles in our in vitro setting. Therefore, triplicate samples of fresh AF medium and SM were analyzed by a mass spectrometry-based untargeted metabolomics approach (TripleTOF, Methods). Combining positive and negative ionization mode, 3092 metabolomic features were detected in total (Methods). From these, $2387(77.20 \%)$ were significantly altered (t-test, p value $<0.05)$ by at least one of the twelve strains (Fig. S6). Hierarchical clustering of the metabolomic feature depletion profiles (i.e. substrates used by the bacteria; Fig. 2A) reflects the phylogenetic relationship between the strains (Fig. 1A). Correlating the phylogenetic distance between the individual strains with the number of shared depleted metabolomic features in AF medium (Fig. S7) showed that phylogenetically similar strains of the consortium have a higher substrate overlap than phylogenetically distant strains $(\mathrm{R}=-0.29, \mathrm{p}=0.017)$. The total number of metabolomic features that are depleted from AF medium greatly varies for the different strains, ranging from over 600 depleted features for M. intestinale YL27 to only 42 for A. muciniphila YL44 (Fig. 2B). The strain specific profiles of depleted metabolomic features were compared pairwise and the number of overlapping features was determined (Fig. 2C). Phylogenetically related strains like E. clostridioformis YL32 and B. coccoides YL58 or M. intestinale YL27 and B. caecimuris I48 share over $50 \%$ of depleted metabolic features each, suggesting a strong substrate overlap in AF medium. Visualizing the extend of overlap between substrate depletion profiles reveals that Bacteroidales, Clostridia and Bacilli strains of the consortium dominate with the highest number of commonly depleted substrates in AF medium (Fig. 2D).

Correlating the growth inhibition in SM ( $\left.\mathrm{d}_{\mathrm{AUC}}\right)$ with the pairwise overlap in depletion profiles (Fig. 2C) revealed that a larger overlap is correlated with a stronger growth inhibition in the corresponding SM ( $\mathrm{R}=-0.46, \mathrm{p}=3.1 \mathrm{E}-08$, Fig. S8). This is illustrated by A. muciniphila YL44, which used only a low numbers of substrates from the AF medium (Fig. 2B) and the SM of which had only little effect on the growth of the other strains of the consortium (Fig. 1C). On the other hand, the strain's growth itself was strongly reduced in the SM of most other consortium members (Fig. 1C, Fig. S2), which depleted a large spectrum of metabolomic features including those used by A. muciniphila YL44 (Fig. 2C).

\section{Genome-informed metabolic potential of the $\mathrm{OMM}^{\mathbf{1 2}}$ consortium}

To be able to infer metabolic interactions between the individual consortium members, a reference dataset giving insight into the metabolic potential of the $\mathrm{OMM}^{12}$ based on genetic information was generated. We screened the genomes of the twelve strains for key enzymes of central carbon metabolism (e.g., fermentation pathways, respiration and amino acid metabolism) (Fig. 3A), as well as for transporters (ABC-transporters and PTS-systems) for carbohydrates and amino acids (Fig. S9, SI data table). Hierarchical clustering of the genomeinformed metabolic potential (Fig. 3A) reflected phylogenetic relationships in several instances, e.g., the Lachnospirales strains of the consortium E. clostridioformis YL32 and B. coccoides YL58, as well as the Oscillospirales strains F. plautii YL31 and A. muris KB18 were found to cluster closely together. Of note, the metabolic potential of T. muris YL45 (Sutterellaceae) was very distinct, clustering differently from all other strains. Generally, high diversity of central and fermentation pathways was found among the consortium members. 
204 Moreover, enzymes for the degradation of monosaccharides (e.g., arabinose, xylose and ribose)

205 and amino acids (e.g., methionine and glutamine) are highly prevalent among consortium 206 members. Phosphotransferase systems were especially prevalent among strains E. faecalis $207 \mathrm{KB1}$, E. clostridioformis YL32 and C. innocuum I46, while ABC transporters for 208 carbohydrates and amino acids were more distributed among all consortium members (Fig. S9).

\section{Metabolite production and fermentation pathways of the $\mathrm{OMM}^{12}$ strains in AF medium}

To gain insights into the metabolites and fermentation products produced and consumed by the individual strains of the consortium in the given in vitro conditions, SM were analyzed using different mass spectrometry approaches (Methods, Fig. S10, Fig. S11). Combining experimentally obtained insights with genome-based information on the presence of key enzymes enabled the generation of broad-scale draft metabolic models of the individual $\mathrm{OMM}^{12}$ community members (Fig. 3B-E, Fig. S12, Supplemental Text C).

To confirm that fermentation pathways identified by genomics were active under in vitro conditions, short chain fatty acid (SCFA) production and consumption was analyzed (Fig. S10A). As observed for the SM metabolic profiles (Fig. S6), hierarchical clustering revealed that closely related bacteria showed similar SCFA production and consumption profiles. Both Bacteroidetes strains produced acetic acid, succinic acid as well as branched-chain fatty acids. Both Lachnospiraceae strains generated high amounts of acetic acid. Butyric acid is produced by strains F. plautii YL31 and C. innocuum I46, the latter also being the only strain of the consortium excreting valeric acid and hexanoic acid. Of note, F. plautii YL31 also consumed lysine, indicating the ability to produce butyric acid from lysine, which was supported by the presence of gene coding for lysine aminomutase (EC 5.4.3.2 and EC 5.4.3.3) as well as two of the following genes encoding key enzymes in the pathway: L-erythro-3.5-diaminohexyanoate dehydrogenase (EC 1.4.11) and 3-keto-5-aminohexanoate cleavage enzyme (EC 2.3.1.247).

Formic acid was produced by several strains and consumed by T. muris YL45 and B. coccoides YL58, indicating the ability of formic acid/ $\mathrm{H}_{2}$ oxidation. T. muris YL45 and B. coccoides YL58 both encode genes for a CO dehydrogenase/acetyl-CoA-synthase (EC 1.2.7.4 and EC 2.3.1.169), the key enzyme of the Wood-Ljungdahl pathway (reductive acetyl-CoA pathway). Formic acid can be processed via this pathway to acetyl-CoA. As another prominent example of bacterial fermentation, lactate production was confirmed (Fig. S10B) for E. faecalis KB1, B. animalis YL2, F. plautii YL31, A. muris YL45, C. innocuum I46 and B. caecimuris, all of which harbor genes coding for the enzyme lactate dehydrogenase (EC 1.1.1.27 and EC 1.1.1.28).

\section{9}

By quantifying amino acid levels we could show that Bacteroidetes and Lachnospiraceae strains exhibited similar amino acid depletion and production profiles. In SM of strains $M$. intestinale YL27 and B. caecimuris I48, elevated levels of a diverse range of amino acids including glutamic acid, histidine, methionine, proline and phenylalanine were detected. Lachnospiraceae strains showed increased levels of isoleucine, tryptophan and valine, while alanine was especially depleted by B. coccoides YL58. Other strains of the consortium showed specific depletion of single amino acids, e.g., F. plautii YL31 strongly depleted lysine and glutamic acid, while E. faecalis KB1 depleted serine.

\section{Growth of $\mathrm{OMM}^{12}$ strains in pairwise co-culture}

Next, we performed a set of experiments to characterize strain-strain interactions in the dynamic community-dependent context. We first analyzed direct competition of all strains in pair-wise co-cultures over the course of $72 \mathrm{~h}$, with serial dilutions every $24 \mathrm{~h}$. While growth was 
monitored continuously by OD 600nm, samples for $\mathrm{pH}$ measurements and qPCR analysis were taken every 24h. The growth curves of most co-cultures, as well as supernatant $\mathrm{pH}$ differed from the corresponding strain specific characteristics observed in monoculture (Fig. S13, Fig. 4A). These differences reflect co-culture dynamics, as can be seen from change in relative abundances over time (Fig. 4B, C).

To identify directionality and mode of interaction between the $\mathrm{OMM}^{12}$ strains, we analyzed the relative changes in absolute abundance (16S rRNA gene copies) as a measure of how successful a strain can grow in co-culture relative to monoculture after $72 \mathrm{~h}$. The mean absolute abundance ratio was calculated for every strain in all pairwise co-cultures $\left(\mathrm{r}_{\mathrm{i}, \mathrm{bm}}=\frac{m_{i, c o}(t 72 h)}{m_{i, m o n o}(t 72 h)}\right)$ (Fig. S14, Methods). If absolute abundance of a strain increased significantly in co-culture relative to monoculture $\left(\mathrm{r}_{\mathrm{bm}}>1\right)$, the interaction was categorized as positive $(+)$, if it decreased $\left(\mathrm{r}_{\mathrm{bm}}<1\right)$ the interaction was categorized as negative $(-)$ (t-test comparing the $\mathrm{r}_{\mathrm{bm}}$ of three independent experiments, Fig. S15). If it did not significantly $(p>0.05)$ differ from that in monoculture $\left(\mathrm{r}_{\mathrm{bm}}=1\right)$, the interaction was categorized as neutral $(0)$. By this, we created a coculture interaction matrix (Fig. 5A): the vast majority of the interactions was classified as amensalistic (0/- and -/0, 46 of 66 of interactions). A smaller subset of interactions was either competitive (-/-, 7 of 66 of interactions) or neutral (0/0, 11 of 66 of interactions). No mutualistic interactions $(+/+)$ were observed. However, one example for each, commensalism $(0 /+$ and $+/ 0$ ) and predation (+/- and -/+), were identified.

The extent to which the individual strains altered the growth of other community members in the co-culture differed distinctly. While E. faecalis KB1 and C. innocuum I46 lead to nine negative co-culture outcomes each, A. muciniphila YL44 and A. muris KB18 only impaired growth of one and zero strains, respectively. Simultaneously, both strains are negatively influenced in most co-cultures, with a significantly decreased absolute abundance in ten and eight co-cultures, respectively. Notably, B. coccoides YL58 is involved in five of seven competitive interactions of the consortium. These observations are in line with the outcomes observed in SM experiments, as strongly negative co-culture outcome correspond to a strong inhibition of a strain in the respective SM (Fig. S16).

\section{Community structure of the OMM $\mathbf{O M}^{12}$ consortium}

280 Next, we set out to investigate if interactions found in co-cultures are transferrable to the strains' behavior in the complete $\mathrm{OMM}^{12}$ community. To this end, all twelve OMM strains were simultaneously co-cultured in AF medium and were serially diluted 1:100 every 24h into fresh AF medium. Relative abundance of all strains after $72 \mathrm{~h}$ and 10 days compared to the inoculum was determined by qPCR for ten replicates each in two independent experiments from different inocula (Fig. S17, Fig. S18, Fig. 5B).

While each of the $\mathrm{OMM}^{12}$ members except $E$. faecalis KB1 was outcompeted to a very low relative abundance in at least one pairwise culture (Fig. 5A), the majority (10 out of 12) of the consortium members were able to coexist in the complex community over the course of $72 \mathrm{~h}$ (Fig. S17A) and up to 10 days. (Fig. 5B, Fig. S17B). Replicate communities showed reproducible community structure, even when different inocula were used (Fig. 5B, Fig. S17B) and especially when compared on the order and phylum level (Fig. S18). Communities were dominated by B. coccoides YL58 and E. faecalis KB1, together making up $>50 \%$ of the relative abundance, which corresponds to their dominant role in SM and co-culture experiments (Fig. 1C, 5A). While strains B. animalis YL2 and L. reuteri I49 were not detectable at $72 \mathrm{~h}$ and 10 days in all replicates, $A$. muris KB18 was found in only few of the communities at 10 days (relative abundance $<1 \%$ ).

\section{E. faecalis KB1 strongly impacts overall community composition}


To understand how interactions observed on the pairwise level transfer to the community context, we derived pairwise correlations from the relative abundance data of all communities at day 10 derived from two independent experiments (Fig. S19). The strong negative influence of E. faecalis KB1 on most other strains observed in pairwise co-cultures applied in the community context as well. High relative abundance of $E$. faecalis KB1 linearly correlated with decreased relative abundance of $C$. innocuum I46 $(\mathrm{R}=-0.87$; $\mathrm{p}<0.05)$, supporting the hypothesis of a predatory interaction between $E$. faecalis KB1 and C. innocuum I46 as observed in co-culture experiments (Fig. 5A, D). In order to identify potentially cross-fed metabolites of C. innocuum $\mathrm{I} 46$ to $E$. faecalis $\mathrm{KB} 1$, we mined metabolomic data of SM for features enriched in $C$. innocuum 146 and depleted by $E$. faecalis KB1. Thereby, we identified several compounds including malate, L-methionine, spermidine and methylglyoxal (Fig. 5E). To experimentally support the idea of cross-feeding, we exemplarily tested uptake of ${ }^{14} \mathrm{C}$-malate into intact cells of E. faecalis KB1. To slow the metabolization of this metabolite, all assays were performed at $18^{\circ} \mathrm{C}$. We found a very fast linear uptake of ${ }^{14} \mathrm{C}$-malate by $E$. faecalis $\mathrm{KB} 1$ within the first $60 \mathrm{~s}$, which could explain why malate utilization confers a growth advantage to this strain (Fig. $5 \mathbf{F})$.

314 Finally, we were interested in how the absence of $E$. faecalis KB1 would affect the overall community structure. We generated a 'dropout' community including all strains of the $\mathrm{OMM}^{12}$ consortium except $E$. faecalis $\mathrm{KB} 1\left(\mathrm{OMM}^{11}-\mathrm{KB} 1\right)$. Compositional analysis revealed increased relative abundance of $C$. innocuum I46 and B. animalis YL2 in the OMM ${ }^{11}-\mathrm{KB} 1$ compared to the full $\mathrm{OMM}^{12}$ community (Fig. 5C). In addition, the absolute abundances of strains $B$. animalis YL2, C. innocuum I46 and B. caecimuris I48 were found to increase significantly (ttest, $\mathrm{p}<0.05$ ) in the absence of $E$. faecalis KB1 (Fig. S20). While the increase in abundance of $B$. animalis YL2 and $C$. innocuum I46 may be explained by absent enterocin production by E. faecalis KB1, the increased abundance of B. caecimuris I48 was unexpected. Further, the abundance of F. plautii YL31, E. clostridioformis YL32, A. muciniphila YL44 and T. muris YL45 was found to decrease in the absence of E. faecalis KB1. This indicates either direct positive effects of $E$. faecalis KB1 on these strains or indirect effects that occur through the overall shift in $\mathrm{OMM}^{11}-\mathrm{KB} 1$ community composition compared to the $\mathrm{OMM}^{12}$ consortium.

\section{Discussion}

A central challenge in gut microbiome research is to understand how interactions between the individual microorganisms affect community-level structure and related functions. Bottom-up approaches involving synthetic communities are valuable tools to study these interactions, as they allow to reduce complexity and to enable strain-specific manipulation. Using an in vitro approach, we focused on characteristics and interactions of the $\mathrm{OMM}^{12}$ community and combined monoculture, pairwise and community cultivation of the strains with genome and metabolomics analysis of their SM. Thereby we reveal that the $\mathrm{OMM}^{12}$ community interaction network is shaped by exploitative and interference competition. In particular, E. faecalis KB1, a low-abundant member of the mammalian gut microbiota, was identified as important driver of in vitro community composition by directly or indirectly altering the abundance of several other consortium members. We provide draft metabolic models of the individual $\mathrm{OMM}^{12}$ strains, which will be a valuable tool for mechanistic studies using this synthetic community.

341 Exploitative (i.e. substrate) competition plays a major role in shaping intestinal bacterial 342 communities (35). Understanding the underlying principles of how bacteria compete for 
available nutrients is essential to predict and control community composition. We found that phylogenetically similar strains showed a higher substrate overlap (Fig. S7), which is in accordance with previous studies demonstrating that phylogeny reflects metabolic capabilities of bacteria (36, 37). Furthermore, overlap in substrate depletion profiles was correlated with growth inhibition in the respective SM (Fig. S8). This clearly indicates strong exploitative competition between individual $\mathrm{OMM}^{12}$ strains. In particular $B$. caecimuris I48, E. faecalis $\mathrm{KB1}$, E. clostridioforme YL32 and B. coccoides YL58 were found to consume a high number of substrates (>200), while their SM inhibited growth of the majority of the other community members (Fig. 1C). Of note, $M$. intestinale YL27 and C. innocuum I46, also consumed over 200 substrates each, but inhibited few other strains. This demonstrates that substrate overlap cannot simply predict inhibition in all cases and other mechanisms (i.e. waste product inhibition) play a role in specific cases.

355

Besides substrate competition, a strain's ability to acidify its environment or release an inhibitory factor (e.g. waste products, bacteriocins) can determine if another species can grow in the exhausted medium or not. Several strains, including B. caecimuris I48, B. coccoides YL58, E. faecalis KB1 and C. inocuum I46 acidified the medium during growth in monoculture. However, only for few species, A. muciniphila YL44, B. caecimuris I48 and B. animalis YL2, acidic $\mathrm{pH}$ correlated with reduced growth (Fig. S4). Moreover, the $\mathrm{pH}$ in the full $\mathrm{OMM}^{12}$ community, where most of the strains coexisted, was also acidic (pH of 6.2), suggesting that $\mathrm{pH}$ modification does not play a major role in driving community composition in vitro. Interference competition by bacteriocins is widespread among gut bacterial communities (38). We found that E. faecalis KB1 produces at least one antimicrobial compound that shows activity against five of the Gram-positive $\mathrm{OMM}^{12}$ strains (B. animalis YL2, E. clostridioformis YL32, F. plautii YL31, C. innocuum I46 and L. reuterii I49) (Fig. 1E). E. faecalis harbors genes coding for at least two different enterocins (enterocin L50A/L50B and enterocin O16). Therefore, we hypothesize that some of the inhibitory effects of $E$. faecalis KB1 on those strains can be attributed to enterocin-mediated killing.

E. faecalis is a prevalent but low abundant member of the undisturbed human and animal microbiota. Following antibiotic therapy, the bacterium can dominate the gut and cause bloodstream infection in immunocompromised individuals (39). Understanding how E. faecalis outcompetes/overgrows other gut microorganisms is important in order to intervene with $E$. faecalis domination in the gut. Besides enterocin-mediated killing we found that metabolite cross-feeding seems to contribute to the interaction of $E$. faecalis $\mathrm{KB} 1$ with $C$. innocuum I46 (Fig. 5D; Fig. S10). Based on metabolic profile mining, we hypothesize, that $E$. faecalis KB1 consumes malate, methionine, arginine and serine among other metabolites in co-culture with C. innocuum I46 (Fig. 5E, Fig. S11). Interestingly, a previous study (40) reported that glucosemalate co-metabolism increases growth of $E$. faecalis over glucose consumption alone. In connection with fast uptake rate of ${ }^{14} \mathrm{C}$-malate by E. faecalis $\mathrm{KB} 1$ (Fig. 5F), this suggests that malate cross-feeding may also contribute to E. faecalis KB1 gain in absolute abundance in coculture with $C$. inoccuum I46.

Using batch culture experiments we were able to investigate assembly and dynamics of full $\mathrm{OMM}^{12}$ and $\mathrm{OMM}^{11}-\mathrm{KB} 1$ dropout communities in vitro. A significant increase in B. animalis YL2 and C. innocuum I46 in the community lacking E. faecalis KB1 suggested that enterocinmediated killing also shapes the more complex community (Fig. S20). Notably, in the full $\mathrm{OMM}^{12}$ community, ten of the twelve strains co-existed over ten days. This was remarkable given the high number of negative pairwise interactions in SM and co-culture experiments. Differences between the behavior of strains in pairwise versus complex communities point at higher-order ecological interactions that emerge in the community context. As previously 
shown in other studies, the underlying mechanisms may involve metabolic flexibility or mixed substrate utilization of the strains in the presence of competitors (41), metabolite cross-feeding and lack of waste-product inhibition and overall change in $\mathrm{pH}$ (11) or an excess of provided substrates in the medium.

Following up, it will be important to assess, if the in vitro findings can be translated to the mouse model. Several differences between in vitro and in vivo conditions were noted. While the in vitro community is dominated by B. coccoides YL58 and E. faecalis KB1, mouse communities are dominated by B. caecimuris I48 and A. muciniphila YL44 (Fig. S21)(26). Enrichment of amino acids and glucose in the used culture medium may favor growth of $B$. coccoides YL58 and E. faecalis KB1 in vitro at the expense of bacteria specialized on utilization of mucin and other complex carbohydrates (42, 43). To this end, it will be worthwhile to modify the in vitro conditions to more closely recapitulate the chemical landscape and spatial structure of the gut in future experiments.

Concluding, our study presents a comprehensive in vitro investigation of strain-strain interactions between members of a widely used synthetic intestinal bacterial community. Characterization of the metabolic profile of individual strains of the consortium as well as analyzing their metabolism and community assembly in co-culture revealed E. faecalis KB1 and B. coccoides YL58 to be important drivers of community composition. Drawing on this detailed understanding of in vitro behavior, our results will enable to employ this model for mechanistic in vivo studies. This step-wise approach may ultimately allow accurate description of interaction dynamics of in vivo gut microbial communities and pave the way for targeted manipulation of the microbiome to promote human health. In particular, extending the approach of dropout communities lacking specific strains could help to elucidate the role of individual players in community functions like dietary breakdown, metabolite production and colonization resistance and to identify general principles of how bacterial interaction networks and the corresponding emergence of higher order interactions shape microbiome function. This will enable the design of therapeutic interventions to control microbial community functions by advanced microbiome engineering.

\section{Methods}

\section{Generation of a 16S gene based phylogenetic tree}

The genomes of the twelve strains of the $\mathrm{OMM}^{12}$ consortium (27) were accessed via DDBJ/ENA/GenBank using the following accession numbers: CP022712.1, NHMR02000001NHMR02000002， CP021422.1， CP021421.1， NHMQ01000001-NHMQ01000005, NHTR01000001-NHTR01000016, CP021420.1, NHMP01000001-NHMP01000020, CP022722.1, NHMU01000001-NHMU01000019, NHMT01000001-NHMT01000003, CP022713.1 and annotated using Prokka (default settings) (44). The 16S rRNA sequences of all strains were obtained. These rRNA FASTA sequences were uploaded to the SINA Aligner v1.2.11 (45) to align these sequences with minimum 95\% identity against the SILVA database. By this, a phylogenetic tree based on RAxML (46), GTR Model and Gamma rate model for likelihood was reconstructed. Sequences with less than $90 \%$ identity were rejected. The obtained tree was rooted using midpoint.root() in the phytools package (47) in R and visualized using iTOL online (48).

\section{Genome annotation for predicting genome-informed metabolic potential}


436 The genomes (accessed via DDBJ/ENA/GenBank as stated above) of the twelve strains of the $\mathrm{OMM}^{12}$ consortium were annotated using prodigal (version V2.6.3, default settings) (49) and KEGG orthologies (KO) for the protein-coding genes were obtained using the tool KOfamscan (default settings) (50). The tool provided multiple KO annotations for each gene with corresponding e-values and threshold scores. In order to get one $\mathrm{KO}$ annotation per gene, the annotation was considered only if a) the internal threshold score was reached (marked '*' by kofamscan) or b) an evalue of $>1 \mathrm{e}-03$ was reached. The remaining annotations were ignored.

\section{Strains and culture conditions}

444 Bacterial cultures were prepared from frozen monoculture stocks in a $10 \mathrm{ml}$ culture and subculture in cell culture flasks (flask T25, Sarstedt) previous to all experiments. Cultures were incubated at $37^{\circ} \mathrm{C}$ without shaking under strictly anaerobic conditions (gas atmosphere $7 \% \mathrm{H}_{2}$, $10 \% \mathrm{CO}_{2}, 83 \% \mathrm{~N}_{2}$ ). All experiments were carried out using AF medium (18 g.l $\mathrm{l}^{-1}$ brain-heart infusion, 15 g. $\mathrm{l}^{-1}$ trypticase soy broth, 5 g.l $\mathrm{l}^{-1}$ yeast extract, 2.5 g. $\mathrm{l}^{-1} \mathrm{~K}_{2} \mathrm{HPO}_{4}, 1 \mathrm{mg} . \mathrm{l}^{-1}$ haemin, 0.5 g. $\mathrm{l}^{-1}$ D-glucose, 0.5 mg. $\mathrm{l}^{-1}$ menadione, 3\% heat-inactivated fetal calf serum, $0.25 \mathrm{~g}^{-1} \mathrm{l}^{-1}$ cysteine- $\mathrm{HCl} \cdot \mathrm{H}_{2} \mathrm{O}$ ). The following strains were used in this study: Enterococcus faecalis $\mathrm{KB} 1$ (DSM32036), Bifidobacterium animalis YL2 (DSM26074), Acutalibacter muris KB18 (DSM26090), Muribaculum intestinale YL27 (DSM28989), Flavonifractor plautii YL31 (DSM26117), Enterocloster clostridioformis YL32 (DSM26114), Akkermansia muciniphila YL44 (DSM26127), Turicimonas muris YL45 (DSM26109), Clostridium innocuum I46 (DSM26113), Bacteroides caecimuris I48 (DSM26085), Limosilactobacillus reuteri I49 (DSM32035), Blautia coccoides YL58 (DSM26115).

\section{Growth measurements}

458 Bacterial growth was measured in 96well round bottom plates (Nunc) using a GenTech Epoch2 plate reader. Inocula were prepared from a previous culture and subculture and diluted in fresh AF medium to $0.01 \mathrm{OD}_{600 \mathrm{nn}}$. Absorption at wavelength $600 \mathrm{~nm}$ was determined in a reaction volume of $100 \mu \mathrm{l}$ in monoculture and SM experiments and $150 \mu \mathrm{l}$ in co-culture experiments. During continuous measurements, the plate was heated inside the reader to $37^{\circ} \mathrm{C}$ and a 30 second double orbital shaking step was performed prior to every measurement.

\section{Generation of spent culture media}

Bacterial cultures and subcultures were grown for 24 hours each in $10 \mathrm{ml}$ AF medium at $37^{\circ} \mathrm{C}$ under anaerobic conditions without shaking. Bacterial spent culture supernatants (SM) were generated by centrifugation of the densely grown subculture at $4^{\circ} \mathrm{C}$ for $20 \mathrm{~min}$ at $5000 \mathrm{xg}$ and subsequent $\mathrm{pH}$ measurement and filter-sterilization $(0.22 \mu \mathrm{m})$. SM samples were aliquoted and immediately frozen at $-80^{\circ} \mathrm{C}$. Samples were thawed under anaerobic conditions previous to growth measurements. Growth of all bacterial monocultures in the spent culture media (SM) of all respective other strains was then measured as described above. SM were inoculated with bacterial monocultures with starting $\mathrm{OD}_{600 \mathrm{~nm}} 0.01$. After monoculture growth of $20 \mathrm{~h}$ in the respective SM (resulting in double spent media, DSM), $\mathrm{pH}$ values were determined.

$\mathrm{pH}$ measurements of bacterial supernatants were performed using a refillable, glass double junction electrode (Orion ${ }^{\mathrm{TM}}$ PerpHecT ${ }^{\mathrm{TM}}$ ROSS $^{\mathrm{TM}}$, Thermo Scientific).

\section{Metabolic profiling of late stationary phase bacterial supernatants}

478 The untargeted analysis was performed using a Nexera UHPLC system (Shimadzu) coupled to a Q-TOF mass spectrometer (TripleTOF 6600, AB Sciex). Separation of the spent media was performed using a UPLC BEH Amide 2.1x100, $1.7 \mu \mathrm{m}$ analytic column (Waters Corp.) with $400 \mu \mathrm{L} / \mathrm{min}$ flow rate. The mobile phase was $5 \mathrm{mM}$ ammonium acetate in water (eluent $\mathrm{A}$ ) and 
$5 \mathrm{mM}$ ammonium acetate in acetonitrile/water (95/5, v/v) (eluent B). The gradient profile was $100 \%$ B from 0 to $1.5 \mathrm{~min}, 60 \% \mathrm{~B}$ at $8 \mathrm{~min}$ and $20 \% \mathrm{~B}$ at $10 \mathrm{~min}$ to $11.5 \mathrm{~min}$ and $100 \% \mathrm{~B}$ at 12 to $15 \mathrm{~min}$. A volume of $5 \mu \mathrm{L}$ per sample was injected. The autosampler was cooled to $10^{\circ} \mathrm{C}$ and the column oven heated to $40^{\circ} \mathrm{C}$. Every tenth run a quality control (QC) sample which was pooled from all samples was injected. The spent media samples were measured in a randomized order. The samples have been measured in Information Dependent Acquisition (IDA) mode. MS settings in the positive mode were as follows: Gas 155 , Gas 2 65, Curtain gas 35, Temperature $500^{\circ} \mathrm{C}$, Ion Spray Voltage 5500, declustering potential 80 . The mass range of the TOF MS and MS/MS scans were $50-2000 \mathrm{~m} / \mathrm{z}$ and the collision energy was ramped from 15 - 55 V. MS settings in the negative mode were as follows: Gas 1 55, Gas 2 65, Cur 35, Temperature $500^{\circ} \mathrm{C}$, Ion Spray Voltage -4500 , declustering potential -80 . The mass range of the TOF MS and MS/MS scans were $50-2000 \mathrm{~m} / \mathrm{z}$ and the collision energy was ramped from $-15--55 \mathrm{~V}$.

The "msconvert" from ProteoWizard (51) were used to convert raw files to mzXML (de-noised by centroid peaks). The bioconductor/R package xcms (52) was used for data processing and feature identification. More specifically, the matched filter algorithm was used to identify peaks (full width at half maximum set to 7.5 seconds). Then the peaks were grouped into features using the "peak density" method (52). The area under the peaks was integrated to represent the abundance of features. The retention time was adjusted based on the peak groups presented in most of the samples. To annotate possible metabolites to identified features, the exact mass and MS2 fragmentation pattern of the measured features were compared to the records in HMBD (53) and the public MS/MS database in MSDIAL (54), referred to as MS1 and MS2 annotation, respectively. The QC samples were used to control and remove the potential batch effect, t-test was used to compare the features' intensity from spent media with fresh media.

507

508

\section{Targeted short chain fatty acid (SCFA) measurement}

The 3-NPH method was used for the quantitation of SCFAs (55). Briefly, $40 \mu \mathrm{L}$ of the SM and $15 \mu \mathrm{L}$ of isotopically labeled standards (ca $50 \mu \mathrm{M}$ ) were mixed with $20 \mu \mathrm{L} 120 \mathrm{mM}$ EDC HCl$6 \%$ pyridine-solution and $20 \mu \mathrm{L}$ of $200 \mathrm{mM} 3-\mathrm{NPH}$ HCL solution. After $30 \mathrm{~min}$ at $40^{\circ} \mathrm{C}$ and shaking at $1000 \mathrm{rpm}$ using an Eppendorf Thermomix (Eppendorf, Hamburg, Germany), 900 $\mu \mathrm{L}$ acetonitrile/water (50/50, v/v) was added. After centrifugation at $13000 \mathrm{U} / \mathrm{min}$ for $2 \mathrm{~min}$ the clear supernatant was used for analysis. The same system as described above was used. The electrospray voltage was set to $-4500 \mathrm{~V}$, curtain gas to $35 \mathrm{psi}$, ion source gas 1 to 55 , ion source gas 2 to 65 and the temperature to $500^{\circ} \mathrm{C}$. The MRM-parameters were optimized using commercially available standards for the SCFAs. The chromatographic separation was performed on a $100 \times 2.1 \mathrm{~mm}, 100 \AA$, $1.7 \mu \mathrm{m}$, Kinetex C18 column (Phenomenex, Aschaffenburg, Germany) column with $0.1 \%$ formic acid (eluent A) and $0.1 \%$ formic acid in acetonitrile (eluent B) as elution solvents. An injection volume of $1 \mu \mathrm{L}$ and a flow rate of 0.4 $\mathrm{mL} / \mathrm{min}$ was used. The gradient elution started at $23 \% \mathrm{~B}$ which was held for $3 \mathrm{~min}$, afterward the concentration was increased to $30 \% \mathrm{~B}$ at $4 \mathrm{~min}$, with another increase to $40 \% \mathrm{~B}$ at $6.5 \mathrm{~min}$, at $7 \mathrm{~min} 100 \%$ B was used which was held for $1 \mathrm{~min}$, at $8.5 \mathrm{~min}$ the column was equilibrated at starting conditions. The column oven was set to $40^{\circ} \mathrm{C}$ and the autosampler to $15^{\circ} \mathrm{C}$. Data acquisition and instrumental control were performed with Analyst 1.7 software (Sciex, Darmstadt, Germany).

\section{Dynamic metabolic profiling of bacterial supernatants}

530 All chemicals were purchased from Sigma Aldrich at the highest purity available. $50 \mu \mathrm{l}$ of the 
internal standards, afterwards the samples were dried under a gentle stream of nitrogen. For derivatization $100 \mu \mathrm{l}$ of a methoxyamine hydrochloride solution $(10 \mathrm{mg} / 1 \mathrm{ml}$ pyridine) were added and the sample was shaken at $40^{\circ} \mathrm{C}$ for $90 \mathrm{~min}$. Afterwards $100 \mu \mathrm{l}$ of MTBSTFA (N(tert-butyldimethyl-silyl)-N-methyl-trifluoroacetamide containing $1 \%$ tert-butyl-dimethylsilylchlorid) was added and the sample was heated at $70^{\circ} \mathrm{C}$ for $45 \mathrm{~min}$. GC-MS-analysis was performed with a QP2010 Plus or Ultra gas chromatograph/mass spectrometer (Shimadzu) equipped with a fused silica capillary column (Equity TM-5; $30 \mathrm{~m} \times 0.25 \mathrm{~mm}, 0.25 \mu \mathrm{m}$ film thickness; SUPELCO) and a quadrupole detector working with electron impact ionization at $70 \mathrm{eV}$. An aliquot of the derivatized samples was injected in 1:5 split mode at an interface temperature of $260^{\circ} \mathrm{C}$ and a helium inlet pressure of $70 \mathrm{kPa}$. After sample injection, the column was first kept at $60^{\circ} \mathrm{C}$ for $3 \mathrm{~min}$ and then developed with a temperature gradient of $10^{\circ} \mathrm{C} \mathrm{min}{ }^{-1}$ to a final temperature of $300^{\circ} \mathrm{C}$. This temperature was held for further $3 \mathrm{~min}$.

544 Pyruvate results were calculated relative to the pyruvate- ${ }^{13} \mathrm{C}_{3}$ standard $\left(\mathrm{R}_{t} 12.2 \mathrm{~min}\right)$, whereas all other metabolites were calculated relative to norvaline $\left(\mathrm{R}_{\mathrm{t}} 17.7 \mathrm{~min}\right)$.

For qualitative sugar analysis $50 \mu \mathrm{l}$ of the medium were dried under a gentle stream of nitrogen. For derivatization $100 \mu \mathrm{l}$ of a methoxyamine hydrochloride solution (10mg/1 ml pyridine) were added and the sample was shaken at $40^{\circ} \mathrm{C}$ for $90 \mathrm{~min}$. Afterwards $100 \mu \mathrm{l}$ of MSTFA (N-methyl$\mathrm{N}$ (trimethylsilyl)trifluoroacetamide containing 1\% trimethylchlorosilane) was added and the sample was heated at $50^{\circ} \mathrm{C}$ for $45 \mathrm{~min}$. GC-MS-analysis was performed as described above. Glucose, fructose, galactose, mannose and trehalose were confirmed with standard solutions.

553 Bacterial cultures and subcultures were grown for 24 hours each in $10 \mathrm{ml} \mathrm{AF}$ medium at $37^{\circ} \mathrm{C}$ under anaerobic conditions without shaking. Monocultures were diluted to $\mathrm{OD}_{600 \mathrm{~nm}} 0.1$ in fresh AF medium. To generate a dense bacterial lawn, monoculture inocula were diluted in LB soft agar to $\mathrm{OD}_{600 \mathrm{~nm}} 0.01$ and poured on a AF medium agar plate. After drying all respective other bacteria were spotted onto the bacterial lawn in duplicates in a volume of $5 \mu$ l with OD $_{600 \mathrm{~nm}}$ 0.1 . Plates were incubated at $37^{\circ} \mathrm{C}$ for $24 \mathrm{~h}$ under anaerobic conditions.

560 Monoculture inocula were prepared from a previous culture and subculture and were diluted to $\mathrm{OD}_{600 \mathrm{~nm}} 0.1$ in fresh AF medium. Following, pairwise co-cultures were generated by pooling diluted inocula in a 1:1 ratio. From each co-culture $150 \mu$ were set aside for $\mathrm{pH}$ measurements and determination of initial relative abundances (timepoint $0 \mathrm{~h}$ ). The remaining co-cultures were diluted 1:10 to $\mathrm{OD}_{600 \mathrm{~nm}} 0.01$ and pipetted into a round bottom 96-well plate (Nunc). Growth measurements were performed as described above for 72 hours. Samples for qPCR analysis and $\mathrm{pH}$ measurements were taken every 24 hours and the co-cultures were serially diluted 1:100 into $150 \mu \mathrm{l}$ fresh AF medium in a new 96-well round bottom plate to allow communities to approach a steady state composition over $\sim 25$ bacterial generations.

\section{DNA extraction}

570 DNA extraction was performed in the 96-well format using the PureLink ${ }^{\mathrm{TM}}$ Pro 96 genomic DNA Kit (Invitrogen) following the corresponding lysis protocol for Gram positive bacterial cells using lysozyme and proteinase $\mathrm{K}$.

\section{Quantitative PCR of bacterial 16S rRNA genes}

574 Quantitative PCR was performed as described previously (23). Strain-specific 16S rRNA 575 primers and hydrolysis probes were used for amplification. Standard curves were determined 576 using linearized plasmids containing the 16S rRNA gene sequence of the individual strains. 
577 The standard specific efficiency was then used for absolute quantification of 16S rRNA gene

578 copy numbers of individual strains.

\section{Determination of co-culture outcomes}

580 Quantitative 16S rRNA copy numbers from the measurement endpoint of three independent co-culture experiments were determined by qPCR. Co-culture outcomes (positive, neutral or negative) were determined by calculating the individual abundance ratio for each strain in coculture relative to monoculture. Therefore, the strain specific absolute abundance at $72 \mathrm{~h}$ in all pairwise co-cultures was divided by the strain specific absolute abundance at $72 \mathrm{~h}$ in

584

585 monoculture $\left(\mathrm{r}_{\mathrm{i}, \mathrm{bm}}=\frac{m_{i, c o}(t 72 h)}{m_{i, \text { mono }}(t 72 h)}\right)$ for every individual experiment. Following, the mean abundance ratio from all individual experiments ( $\mathrm{n}=3$ per strain combination) was calculated. Significance was determined using a two-sided t-test.

\section{Community experiments}

589 Monoculture inocula were prepared from a previous culture and subculture and were diluted to $\mathrm{OD}_{600 \mathrm{~nm}} 0.1$ in fresh AF medium. Following, the community inoculum with equivalent ratios of all 12 strains was generated from this dilution. The inoculum was distributed to ten cell culture flasks, thereby diluting the inoculum 1:10 to $10 \mathrm{ml}$ fresh AF medium, resulting in a starting $\mathrm{OD}_{600 \mathrm{~nm}} 0.01$. Cell culture flasks were incubated at $37^{\circ} \mathrm{C}$ without shaking under anaerobic conditions. Every 24h for 10 days samples were taken for qPCR analysis, OD measurement and $\mathrm{pH}$ measurement and cultures were diluted 1:100 in 10ml fresh-AF medium.

\section{Malate uptake measurements}

Uptake of ${ }^{14} \mathrm{C}$-malate by $E$. faecalis $\mathrm{KB} 1$ was determined in principle as previously described (56). Briefly, E. faecalis was grown anaerobically in LB medium with $40 \mathrm{mM}$ malate and harvested in mid-log phase. Cells were centrifuged, washed twice with $50 \mathrm{mM}$ Tris-HCl buffer (pH 7.4) containing $10 \mathrm{mM} \mathrm{MgCl}_{2}$ and resuspended in $50 \mathrm{mM}$ Tris-maleate buffer ( $\mathrm{pH} \mathrm{7.2)}$ containing $5 \mathrm{mM} \mathrm{MgCl}_{2}$, thereby adjusting the cell suspension to an $\mathrm{OD}_{600}$ of 10 . For transport

602

603 assays, this cell suspension was diluted $1: 10$ with $50 \mathrm{mM}$ Tris-maleate buffer $(\mathrm{pH} 7.2)$ containing $5 \mathrm{mM} \mathrm{MgCl}_{2}$ and $1 \%$ (w/v) peptone. Uptake of ${ }^{14} \mathrm{C}$-malate $\left(55 \mathrm{mCi}^{\circ} \mathrm{mmol}^{-1}\right.$ [Biotrend]) was measured at a total substrate concentration of $10 \mu \mathrm{M}$ at $18^{\circ} \mathrm{C}$. At various time intervals, transport was terminated by the addition of stop buffer $(100 \mathrm{mM}$ potassium phosphate buffer, $\mathrm{pH}$ 6.0, $100 \mathrm{mM} \mathrm{LiCl}$ ), followed by rapid filtration through membrane filters (MN gf$50.4 \mu \mathrm{m}$; Macherey-Nagel). The filters were dissolved in $5 \mathrm{ml}$ of scintillation fluid (MP Biomedicals), and radioactivity was determined in a liquid scintillation analyzer (PerkinElmer). Total protein content of E. faecalis cells in relation to $\mathrm{OD}_{600}$ was determined

\section{Data analysis and Figures}

613 Data was analyzed using R Studio (Version 1.4.1103). Heatmaps were generated using the $\mathrm{R}$ 614 pheatmap package (https://github.com/raivokolde/pheatmap). Plots were generated using the 615 R ggplot2 package (58) and ggpubR package (https://github.com/kassambara/ggpubr). Figures 616 were partly generated using BioRender (https://biorender.com) and Adobe Illustrator CC 617 (Adobe Inc.). 


\section{Figure Legends}

\section{Figure 1: Growth analysis of $\mathbf{O M M}^{12}$ strains in spent media experiments}

(A) Phylogenetic tree for bacteria of the $\mathrm{OMM}^{12}$ consortium based on the individual 16S rRNA genes. The consortium represents the five major phyla of the murine gastrointestinal tract: Firmicutes (green), Bacteroidetes (orange), Verrucomicrobia (purple), Actinobacteria (blue) and Proteobacteria (red). (B) Flowchart depicting spent culture medium (SM) preparation by growing bacterial monocultures in fresh AF medium for 20h. Culture supernatants were sterilefiltered, samples for $\mathrm{pH}$ measurements and MS were collected, and the SM was used as culture medium for the growth of all respective other strains. After growth of the individual strains in the specific SM, pH of the double spent medium (DSM) was determined. Differences in $\mathrm{pH}$ were then analyzed by calculating the corresponding $\Delta \mathrm{pH}_{\mathrm{SM}}$ and $\Delta \mathrm{pH}_{\mathrm{DSM}}$. (C) Monoculture growth in SM resulted in mostly decreased area under the growth curve (AUC) values in comparison to fresh AF medium, which was analyzed by calculating the inhibition factor $\mathrm{d}_{\mathrm{AUC}}$. $\mathrm{d}_{\text {AUC }}$ was calculated from the mean AUC of three independent experiments relative to the mean AUC in fresh medium. (D) The mean pH of all SM (center of circles) and DSM (outer tiles) after growth of the individual strains in fresh medium and the respective SM was determined from three independent experiments. (E) Spot assays to determine production of antibacterial agents. All bacterial strains of the $\mathrm{OMM}^{12}$ consortium were spotted onto a bacterial lawn of all the respective other strains. Inhibition zones were observed for B. animalis YL2, F. plautii YL31, E. clostridioformis YL32, C. innocuum I46 and L. reuteri I49 when E. faecalis KB1 was spotted. No inhibition zone was seen for $E$. faecalis KB1 on itself. AF medium with $E$. faecalis KB1 spotted is shown as control.

\section{Figure 2: Overlap of substrate depletion profiles between individual $\mathrm{OMM}^{12}$ strains}

(A) Depletion profiles of substrates after bacterial growth to stationary phase in AF medium were determined by untargeted MS from three independent experiments. All metabolomic features (rows) that significantly decreased ( $\mathrm{p}<0.05$ compared to fresh media) compared to fresh medium for at least one of the twelve strains are shown in red. Dark-red indicates strong depletion, while white indicates no depletion of the metabolomics feature. Hierarchical clustering of strain specific profiles as well as metabolomic features reveal profile similarities between phylogenetically similar strains. (B) Bar plot showing the total number of significantly ( $p<0.05$ compared to fresh media) depleted metabolomic features in AF medium for the individual strains. (C) Pairwise overlap in depletion profiles in percent of the total number of individually depleted metabolomic features. (D) Euler diagram depicting total number of depleted metabolomic features and overlap within the consortium. Size of the ellipse denotes number of depleted features, size of overlap between ellipses denotes number of features that are shared when comparing the individual profiles.

Figure 3: Metabolic potential of the $\mathrm{OMM}^{12}$ strains

(A) $\mathrm{OMM}^{12}$ genomes were screened for a hand-curated set of key enzymes to determine the strains' potential to use a diverse range specific substrates, metabolic pathways and release fermentation end products. A potential substrate and pathway utilization was considered positive (green) if one of the associated KO's was found in the respective genome (SI data table). Consequently, positive hits do not indicate completeness of the pathway. If none of the associated KO's was found in the respective genome, the potential substrate and pathway utilization was considered negative (grey). Metabolites and pathways were sorted by functional 
665

666

667

668

669

670

671

672

\section{3}

674

675

676

677

678

679

680

681

682

683

684

685

686

687

688

689

\section{0}

691

692

693

694

695

696

697

698

699

700

701

702

703

704

705

706

707

708

709

710

711

groups. (B) By combining metabolomics data (MS, Fig. S10, S11) with genome-based information on the presence of key enzymes, broad-scale draft metabolic models of the individual $\mathrm{OMM}^{12}$ strains were generated (supplementary text C, Fig. S12). Here, the models for strains E. faecalis KB1, (C) B. caecimuris I48, (D) B. coccoides L58 and (E) F. plautii YL31 are shown. Models of remaining strains of the consortium are shown in Fig. S12. Experimentally confirmed substrates, products or enzymes are shown in black. Hypothetical substrates, products or enzymes are shown in grey.

\section{Figure 4: Pairwise cultures of the $\mathrm{OMM}^{12}$ strains}

(A) $\mathrm{OMM}^{12}$ pairwise strain combinations (12 monocultures, 66 co-cultures) were cultured in a 1:1 ratio in fresh AF medium over the course of 72 hours and growth, $\mathrm{pH}$ and relative abundance was monitored over time in three independent experiments. Growth at $\mathrm{OD}_{600 \mathrm{~nm}}$ and $\mathrm{pH}$ is shown as mean with the corresponding standard deviation (grey), relative abundance over time is shown exemplary for one of the three experiments. Examples of how growth curves develop with changing relative abundances is shown for the co-culture of T. muris YL45 and L. reuteri $\mathrm{I} 49(\mathbf{B})$. Starting with a $\mathrm{OD}_{600 \mathrm{~nm}}$ ratio of approximately $1: 1$, final mean $\mathrm{OD}_{600 \mathrm{~nm}}$ values after the first two turnovers are low, corresponding to YL45 dominating the co-culture. After 48h, L. reuteri I49 resumes growth and final OD values increase. With L reuteri I49 dominating the community in the end, the growth curve as well resembles L. reuteri I49 monoculture growth. Similarly, $\mathrm{pH}$ values reflect changes in co-culture structure, as can be observed e.g. in the co-culture of C. innoccum I46 and B. coccoides YL58 (C). In monoculture, C. innocuum I46 does not strongly acidify its environment, while YL58 acidifies the culture supernatant to around $\mathrm{pH}$ 6.0. In accordance with this observation, $\mathrm{pH}$ values of the co-culture supernatant only drop with increasing dominance of YL58 in the co-culture.

\section{Figure 5: Transferring pairwise interactions to the community level}

(A) By comparing the mean bacterial abundance from three independent experiments in coculture to the mean abundance in monoculture, a pairwise interaction matrix was generated. Interactions where the individual abundance in co-culture significantly (t-test, $\mathrm{p}<0.05$ ) increased are indicated with $(+)$, interactions where it significantly decreased are indicated with $(-)$ and interaction where the abundance did not change in comparison to monoculture growth were indicated with (0). (B) Using a serial passaging batch culture setup, the OMM ${ }^{12}$ community composition was analyzed after ten days of serial dilutions by comparing the relative strain abundances of ten replicates (F1-F10) with the inoculum. (C) To study the influence of $E$. faecalis $\mathrm{KB} 1$ on community composition, a $\mathrm{OMM}^{11}-\mathrm{KB} 1$ dropout community was constructed and relative abundance at day ten was analyzed for ten replicates (F1-F10). (D) Based on SM experiments, pairwise co-culture and community experiments a predation interaction mechanism between strains $C$. innocuum I46 and E. faecalis KB1 is proposed. We hypothesize, that KB1 produces a bacteriocin inhibiting I46, and benefits from cross-feeding on I46 derived metabolites. (E) Potentially cross-fed metabolites were determined by comparing SM profiles (determined by untargeted MS) of KB1 and I46 for metabolites that are strongly produced by I46 and consumed by KB1. Verified annotations are shown in green, potential annotations are shown in black and not annotated compounds are shown in grey as the corresponding feature identification numbers. (F) Time course of malate uptake by whole cells of $E$. faecalis $\mathrm{KB} 1$. Rates of ${ }^{14} \mathrm{C}$-malate uptake were measured at a final malate concentration of $10 \mu \mathrm{M}$ at $18^{\circ} \mathrm{C}$. Standard deviations are estimated from three biological replicates. 


\section{Acknowledgements}

713 The authors thank D. Ring, C. Beck and C. Schwarz for their technical support and members

714 of the Stecher laboratory for helpful feedback and discussions. This research received funding

715 by the German Research Foundation (DFG, German Research Foundation, Projektnummer

716 395357507- SFB 1371, Projektnummer 279971426 and 315980449), the European Research

717 Council (ERC) under the European Union's Horizon 2020 research and innovation programm

718 (Grant Agreement 865615), the German Center for Infection Research (DZIF) and the Center

719 for Gastrointestinal Microbiome Research (CEGIMIR).

\section{Author contributions}

B.S., A.S., K.J. and A.S.W. conceived and designed the experiments. A.S.W., A.v.S., A.B., A.C.D.R., L.R., S.G., K.K. and C.H. performed the experiments. A.S.W., A.B., A.C.D.R., L.R., C.M. C.H., K.K., S.G. and P.M. analyzed the data. P.M., K.K., C.M., C.H., K.J. and W.E. contributed materials/ analysis tools. B.S. coordinated the project. B.S., L.M.J. and A.S.W. wrote the original draft and all authors reviewed and edited the draft manuscript.

\section{Competing interests}

730 The authors declare no competing financial interests.

\section{References}

1. Gilbert J, Blaser M, Caporaso J, Jansson J, Lynch S, Knight R. Current understanding of the human microbiome. Nat Med. 2018:https://doi.org/10.1038/nm.4517.

737 2. Forster S, Kumar N, Anonye B, Almeida A, Viciani E, Stares M, et al. A human gut bacterial genome and culture collection for improved metagenomic analyses. Nat Biotechnol. 2019:https://doi.org/10.1038/s41587-018-0009-7.

739 3. Blasche S, Kim Y, Mars R, Kafkia E, Maansson M, Machado D, et al. Emergence of stable coexistence in a complex microbial community through metabolic cooperation and spatio-temporal niche partitioning. preprint. 2019:https://doi.org/10.1101/541870

4. Amor D, Ratzke C, Gore J. Transient invaders can induce shifts between alternative stable states of microbial 2020:https://doi.org/10.1126/sciadv.aay8676.

5. Faith J, Guruge J, Charbonneau M, Subramanian S, Seedorf H, Goodman A, et al. The long-term stability of the human gut microbiota. Science. 2013:https://doi.org/10.1126/science.1237439.

748 6. Coyte K, Schluter J, Foster K. The ecology of the microbiome: Networks, competition, and stability. Science. 2015:https://doi.org/10.1126/science.aad2602.

7. Gralka M, Szabo R, Stocker R, Cordero O. Trophic Interactions and the Drivers of Microbial Community Assembly. Current Biology. 2020:https://doi.org/10.1016/j.cub.2020.08.007.

8. Granato E, Meiller-Lagrand T, Foster K. The Evolution and Ecology of Bacterial Warfare. Current Biology. 2019:https://doi.org/10.1016/j.cub.2019.04.024. 
9. Caballero S, Kim S, Carter R, Leiner I, Sušac B, Miller L, et al. Cooperating Commensals Restore Colonization Resistance to Vancomycin-Resistant Enterococcus faecium. Cell Host Microbe. 2017:https://doi.org/10.1016/j.chom.2017.04.002. 10. Gutiérrez N, Garrido D. Species Deletions from Microbiome Consortia Reveal Key Metabolic Interactions between Gut Microbes. mSystems. 2019:https://doi.org/10.1128/mSystems.00185-19.

11. Ratzke C, Barrere J, Gore J. Strength of species interactions determines biodiversity and stability in microbial communities. Nat Ecol Evol. 2020:https://doi.org/10.1038/s41559020-1099-4.

12. Kim S, Covington A, Pamer E. The intestinal microbiota: Antibiotics, colonization resistance, and enteric pathogens. Immunological reviews.

766 2017:https://doi.org/10.1111/imr.12563.

13. Kreuzer M, Hardt W. Affects Colonization Resistance Against Enteropathogenic

768

769 Bacteria. Annual review of microbiology. 2020:https://doi.org/10.1146/annurev-micro020420-13457.

14. Lloyd-Price J, Arze C, Ananthakrishnan A, ..., Huttenhower C. Multi-omics of the gut microbial ecosystem in inflammatory bowel diseases. Nature. 2019:https://doi.org/10.1038/s41586-019-1237-9. design of a microbial consortium of mucosal sugar utilizers reduces Clostridiodes difficile colonization. 2020;Nature Communications:https://doi.org/10.1038/s41467-020-18928-1. 16. Freilich S, Kreimer A, Meilijson I, Gophna U, Sharan R, Ruppin E. The large-scale organization of the bacterial network of ecological co-occurrence interactions. Nucleic Acid Research. 2010:https://doi.org/10.1093/nar/gkq118. 17. Shoaie S, Karlsson F, Mardinoglu A, Nookaew I, Bordel S, Nielsen J. Understanding the interactions between bacteria in the human gut through metabolic modeling. Scientific reports. 2013:https://doi.org/10.1038/srep02532. et al. Quantifying Diet-Induced Metabolic Changes of the Human Gut Microbiome. Cell metabolism. 2015:https://doi.org/10.1016/j.cmet.2015.07.001.

785 19. Biggs M, Medlock G, Moutinho T, Lees H, Swann J, Kolling G, et al. Systems-level 786 metabolism of the altered Schaedler flora, a complete gut microbiota. ISME. 2017:https://doi.org/10.1038/ismej.2016.130.

20. Medlock G, Carey M, McDuffie D, Mundy M, Giallourou N, Swann J, et al. nferring Metabolic Mechanisms of Interaction within a Defined Gut Microbiota. Cell Systems. 2018:https://doi.org/10.1016/j.cels.2018.08.003.

21. Venturelli O, Carr A, Fisher G, Hsu R, Lau R, Bowen B, et al. Deciphering microbial interactions in synthetic human gut microbiome communities. Mol Syst Biol. 2018:https://doi.org/10.15252/msb.20178157.

794 22. Clark R, Connors B, Stevenson D, Hromada S, Hamilton J, Amador-Noguez D, et al. 795 Design of synthetic human gut microbiome assembly and function. bioRxiv. 796 2020: https://doi.org/10.1101/2020.08.19.241315.

797 23. Brugiroux S, Beutler M, Pfann C, Garzetti D, Ruscheweyh H, Ring D, et al. Genome798 guided design of a defined mouse microbiota that confers colonization resistance against 799 Salmonella enterica serovar Typhimurium. Nat Microbiol. 800 2016:https://doi.org/10.1038/nmicrobiol.2016.215.

801 24. Studer N, Desharnais L, Beutler M, Brugiroux S, Terrazos M, Menin L, et al. Functional 802 Intestinal Bile Acid 7 $\alpha$-Dehydroxylation by Clostridium scindens Associated with Protection 803 from Clostridium difficile Infection in a Gnotobiotic Mouse Model. Frontiers in cellular and 804 infection microbiology. 2016:https://doi.org/10.3389/fcimb.2016.00191. 
821

822

823

824

825

826

827

828

829

830

831

832

833

834

835

836

837

838

839

840

841

842

843

844

845

846

847

848

849

850

851

852

853

854

25. Herp S, Brugiroux S, Garzetti D, Ring D, Jochum L, Beutler M, et al. Mucispirillum schaedleri Antagonizes Salmonella Virulence to Protect Mice against Colitis. Cell Host Microbe. 2019:https://doi.org/10.1016/j.chom.2019.03.004.

26. Eberl C, Ring D, Münch P, Beutler M, Basic M, Slack E, et al. Reproducible Colonization of Germ-Free Mice With the Oligo-Mouse-Microbiota in Different Animal Facilities. Front Microbiol. 2020:https://doi.org/10.3389/fmicb.2019.02999.

27. Garzetti D, Brugiroux S, Bunk B, Pukall R, McCoy K, Macpherson A, et al. HighQuality Whole-Genome Sequences of the Oligo-Mouse-Microbiota Bacterial Community. Genome announcements. 2017:https://doi.org/10.1128/genomeA.00758-17.

28. Lagkouvardos I, Pukall R, Abt B, Foesel B, Stecher B, Clavel T. The Mouse Intestinal Bacterial Collection (miBC) provides host-specific insight into cultured diversity and functional potential of the gut microbiota. Nat Microbiol. 2016:https://doi.org/10.1038/nmicrobiol.2016.131.

29. Bolsega S, Basic M, Smoczek A, Buettner M, Eberl C, Ahrens D, et al. Composition of the Intestinal Microbiota Determines the Outcome of Virus-Triggered Colitis in Mice. Frontiers in immunology. 2019:https://doi.org/10.3389/fimmu.2019.01708.

30. Kuczma M, Szurek E, Cebula A, Chassaing B, Jung Y, Kang S, et al. Commensal epitopes drive differentiation of colonic Tregs. Science advances. 2020:https://doi.org/10.1126/sciadv.aaz3186.

31. Nowosad C, Mesin L, Castro T, Wichmann C, Donaldson G, Araki T, et al. Tunable dynamics of $\mathrm{B}$ cell selection in gut germinal centres. Nature. 2020:https://doi.org/10.1038/s41586-020-2865-9.

32. Marion S, Desharnais L, Studer N, Dong Y, Notter M, Poudel S, et al. Biogeography of microbial bile acid transformations along the murine gut. Journal of lipid research. 2020:https://doi.org/10.1194/jlr.RA120001021.

33. Cintas L, Casaus P, Holo H, Hernandez P, Nes I, Håvarstein L. Enterocins L50A and L50B, two novel bacteriocins from Enterococcus faecium L50, are related to staphylococcal hemolysins. J Bacteriol. 1998:https://doi.org/10.1128/JB.180.8.988-994.998.

34. Blin K, Shaw S, Steinke K, Villebro R, Ziemert N, Lee S, et al. antiSMASH 5.0: updates to the secondary metabolite genome mining pipeline. Nucleic Acids Research. 2019:https://doi.org/10.1093/nar/gkz310.

35. Berry D, Widder S. Deciphering microbial interactions and detecting keystone species with co-occurrence networks. Front Microbiol. 2014;5:219.

36. Goberna M, Verdu M. Predicting microbial traits with phylogenies. ISME Journal. 2016: https://doi.org/10.1038/ismej.2015.171.

37. Langille M, Zaneveld J, Caporaso J, McDonald D, Knights D, Reyes J, et al. Predictive functional profiling of microbial communities using 16S rRNA marker gene sequences. Nature biotechnology. 2013:https://doi.org/10.1038/nbt.2676.

38. Cotter P, Ross R, Hill C. Bacteriocins - a viable alternative to antibiotics. Nature reviews Microbiology. 2013:https://doi.org/10.1038/nrmicro2937.

39. Ubeda C, Taur Y, Jenq R, Equinda M, Son T, Samstein M, et al. Vancomycin-resistant Enterococcus domination of intestinal microbiota is enabled by antibiotic treatment in mice and precedes bloodstream invasion in humans. The Journal of clinical investigation. 2020:https://doi.org/10.1172/JCI43918.

40. Mortera P, Espariz M, Suárez C, Repizo G, Deutscher J, Alarcón S, et al. Fine-tuned transcriptional regulation of malate operons in Enterococcus faecalis. Applied and environmental microbiology. 2012:https://doi.org/10.1128/AEM.07280-11.

41. Estrela S, Sanchez-Gorostiaga A, Vila J, Sanchez A. Nutrient dominance governs the assembly of microbial communities in mixed nutrient environments. preprint. 2020:https://doi.org/10.1101/2020.08.06.239897. 
42. Berry D, Stecher B, Schintlmeister A, Reichert J, Brugiroux S, Wild B, et al. Hostcompound foraging by intestinal microbiota revealed by single-cell stable isotope probing. Proc Natl Acad Sci U S A. 2013:https://doi.org/10.1073/pnas.1219247110.

43. Ottman N, Davids M, Suarez-Diez M, Boeren S, Schaap P, Martins Dos Santos V, et al. Genome-Scale Model and Omics Analysis of Metabolic Capacities of Akkermansia muciniphila Reveal a Preferential Mucin-Degrading Lifestyle. Appl Environ Microbiol. 2017:https://doi.org/10.1128/AEM.01014-17.

44. Seeman T. Prokka: rapid prokaryotic genome annotation. Bioinformatics. 2014:https://doi.org/10.1093/bioinformatics/btu153.

45. Pruesse E, Peplies J, Gloeckner F. SINA: Accurate high-throughput multiple sequence alignment of ribosomal RNA genes. Bioinformatics. 2012:https://doi.org/10.1093/bioinformatics/bts252.

46. Stamatakis A. RAxML version 8: a tool for phylogenetic analysis and post-analysis of large phylogenies Bioinformatics. 2014:https://doi.org/10.1093/bioinformatics/btu033.

47. Revell L. phytools: an R package for phylogenetic comparative biology (and other things). Methods in Ecology and Evolution. 2012:https://doi.org/10.1111/j.2041210X.11.00169.X.

48. Letunic I, Bork P. Interactive Tree Of Life (iTOL): an online tool for phylogenetic tree display and annotation. Bioinformatics. 2007:https://doi.org/10.1093/bioinformatics/btl529.

49. Hyatt D, Chen G, Locascio P, Land M, Larimer F, Hauser L. Prodigal: prokaryotic gene recognition and translation initiation site identification. BMC bioinformatics. 2010:https://doi.org/10.1186/471-2105-11-119.

50. Aramaki T, Blanc-Mathieu R, Endo H, Ohkubo K, Kanehisa M, Goto S, et al. KofamKOALA: KEGG Ortholog assignment based on profile HMM and adaptive score threshold. Bioinformatics. 2020:https://doi.org/10.1093/bioinformatics/btz859.

51. Kessner D, Chambers M, Burke R, Agus D, Mallick P. ProteoWizard: open source software for rapid proteomics tools development. Bioinformatics. 2008:https://doi.org/10.1093/bioinformatics/btn323.

52. Smith C, Want E, O'Maille G, Abagyan R, Siuzdak G. XCMS: processing mass spectrometry data for metabolite profiling using nonlinear peak alignment, matching, and identification. Analytical chemistry. 2006:https://doi.org/10.1021/ac051437y.

53. Wishart D, Feunang Y, Marcu A, Guo A, Liang K, ..., et al. HMDB 4.0: the human metabolome database for 2018. Nucleic acids research. 2018:https://doi.org/10.1093/nar/gkx89.

54. Tsugawa H, Cajka T, Kind T, Ma Y, Higgins B, Ikeda K, et al. MS-DIAL: dataindependent MS/MS deconvolution for comprehensive metabolome analysis. Nature methods. 2015:https://doi.org/10.1038/nmeth.3393.

55. Han J, Lin K, Sequeira C, Borchers CH. An isotope-labeled chemical derivatization method for the quantitation of short-chain fatty acids in human feces by liquid chromatography-tandem mass spectrometry. Analytica Chimica Acta. 2015;854:86-94.

56. Mokhtari A, Blancato VS, Repizo GD, Henry C, Pikis A, Bourand A, et al. Enterococcus faecalis utilizes maltose by connecting two incompatible metabolic routes via a novel maltose 6'-phosphate phosphatase (MapP). Mol Microbiol. 2013;88(2):234-53.

57. Bradford MM. A rapid and sensitive method for the quantitation of microgram quantities of protein utilizing the principle of protein-dye binding. Anal Biochem. 1976;72:24854.

58. Wickham H. ggplot2: Elegant graphics for data analysis. Springer, New York. 2016:https://ggplot2.tidyverse.org. 


\section{Figure 1}

A

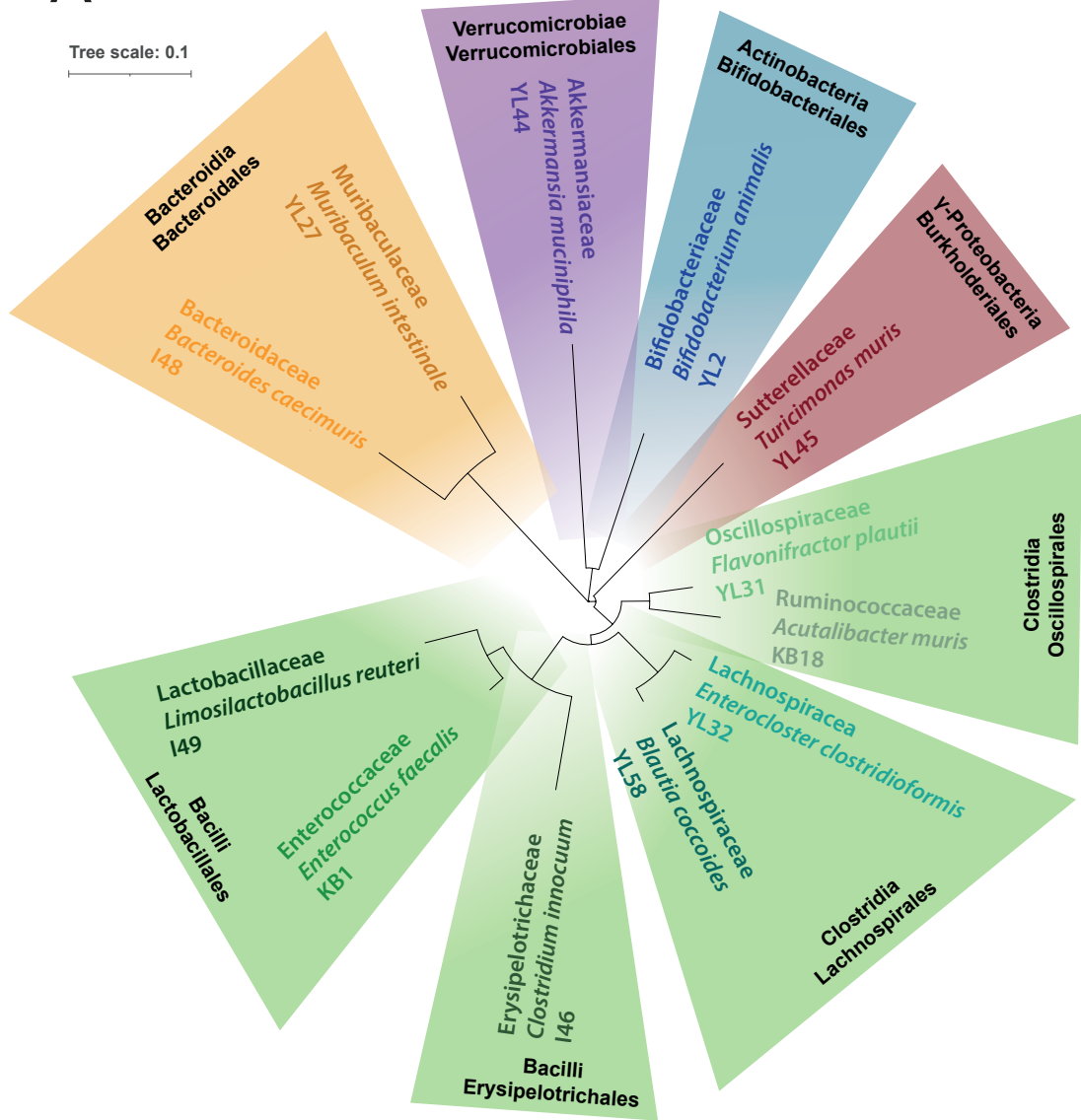

D
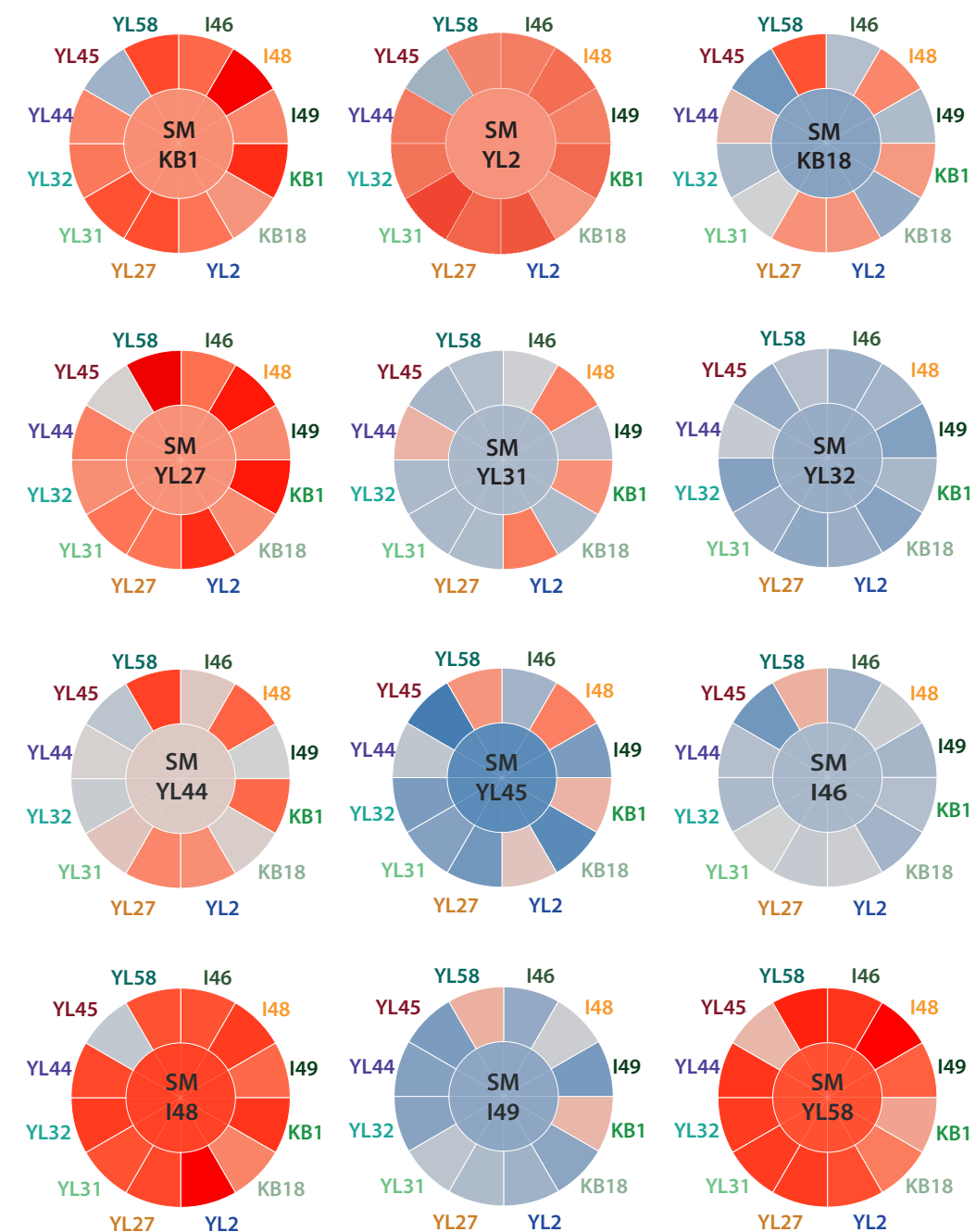

B

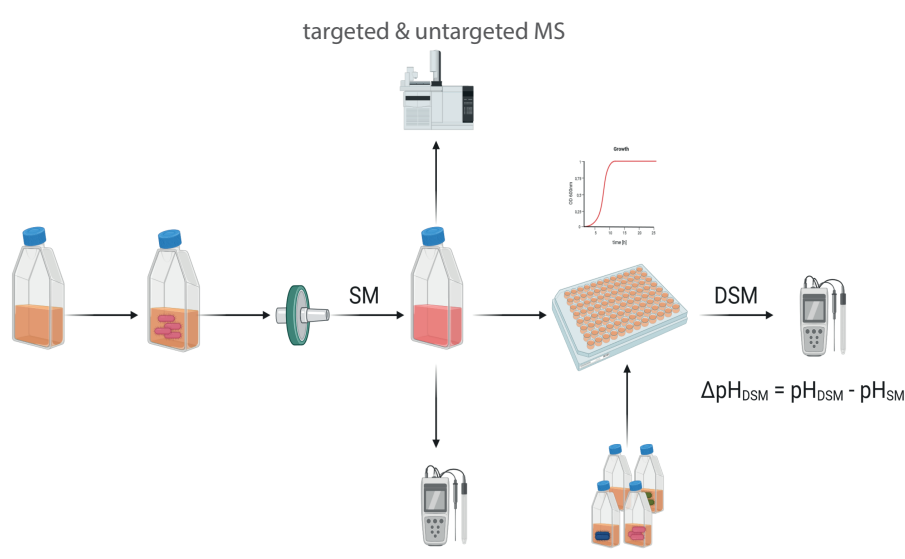

$\Delta \mathrm{pH}_{\mathrm{SM}}=\mathrm{pH}_{\mathrm{SM}}-\mathrm{pH}_{\text {fresh medium }}$
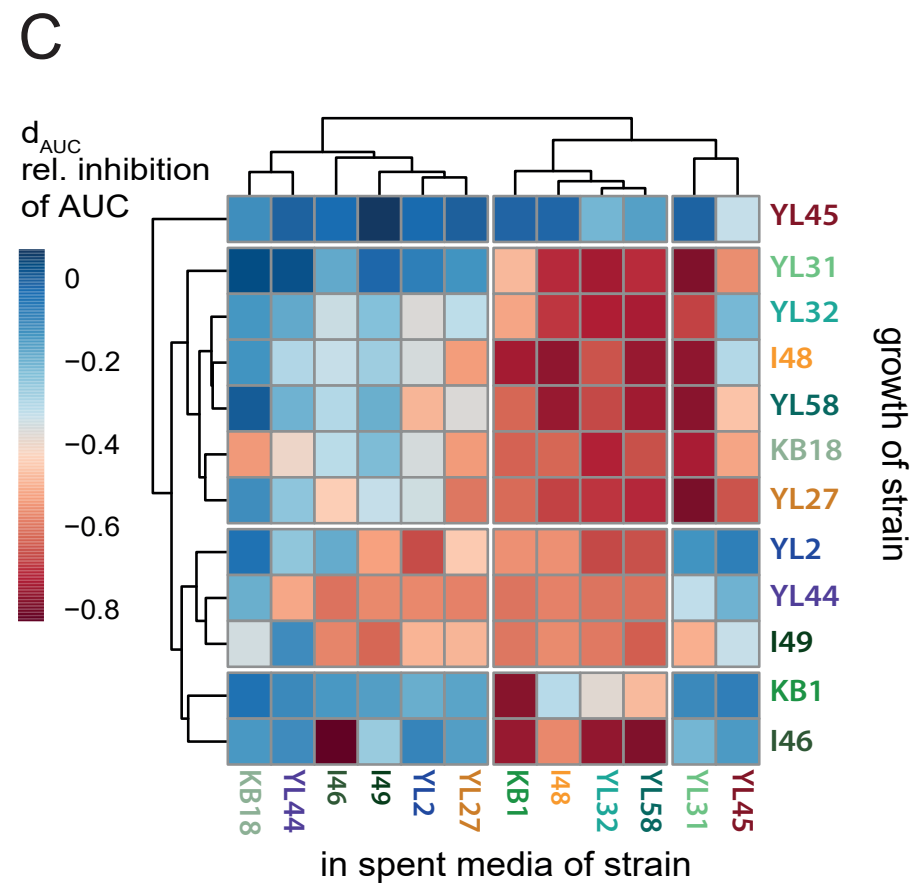

E

Spots of E. faecalis KB1 on:

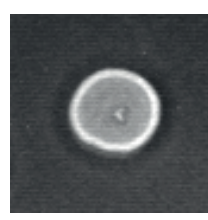

YL2

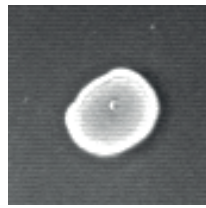

149

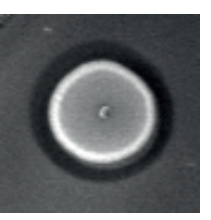

YL31

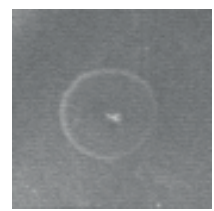

KB1

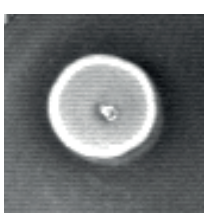

YL32

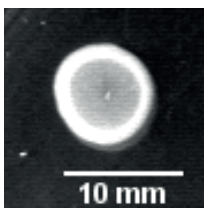

control

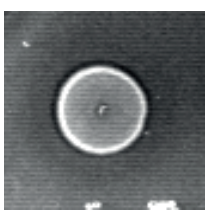

146 
bioRxiv preprint doi: https://doi.org/10.1101/2021.02.25.432904; this version posted February 26, 2021. The copyright holder for this preprint (which was not certified by peer review) is the author/funder. All rights reserved. No reuse allowed without permission.

Figure 2

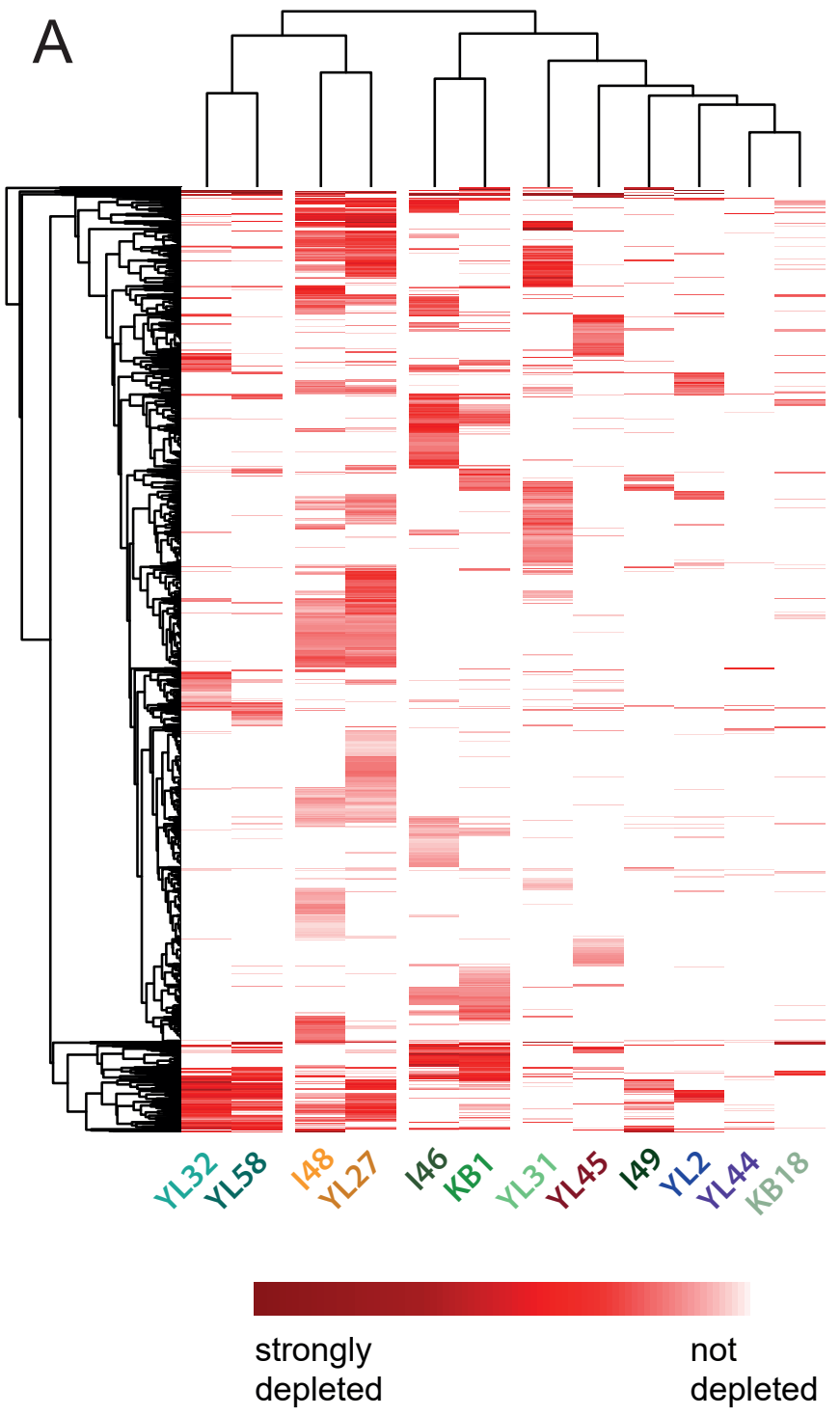

B

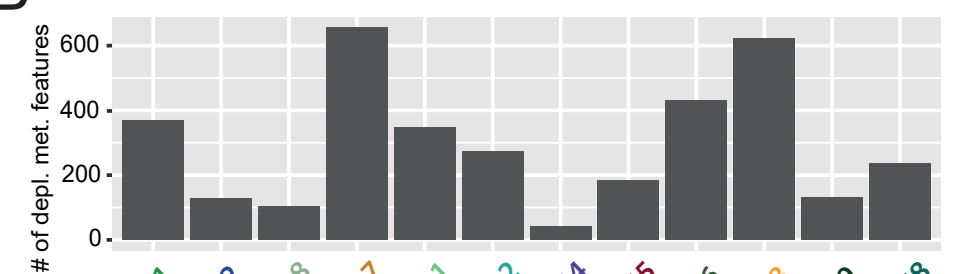

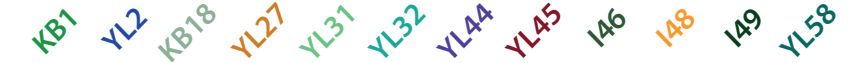

C

\begin{tabular}{|l|l|l|l|l|l|l|l|l|l|l|l|l|l|}
\hline 100.00 & 25.78 & 27.62 & 17.99 & 24.93 & 43.75 & 59.52 & 31.89 & 48.84 & 15.92 & 66.15 & 54.04 & KB1 \\
\hline 8.92 & 100.00 & 27.62 & 11.43 & 15.76 & 16.91 & 23.81 & 5.41 & 8.14 & 12.22 & 23.08 & 20.00 & YL2 \\
\hline 7.84 & 22.66 & 100.00 & 9.76 & 8.02 & 5.88 & 11.90 & 10.81 & 10.93 & 10.93 & 10.00 & 9.36 & KB18 \\
\hline 31.89 & 58.59 & 60.95 & 100.00 & 54.73 & 51.84 & 61.90 & 25.41 & 28.84 & 69.94 & 54.62 & 52.77 & YL27 \\
\hline 23.51 & 42.97 & 26.67 & 29.12 & 100.00 & 28.68 & 30.95 & 13.51 & 14.19 & 27.97 & 36.15 & 22.13 & YL31 \\
\hline 32.16 & 35.94 & 15.24 & 21.49 & 22.35 & 100.00 & 59.52 & 31.89 & 20.70 & 19.77 & 62.31 & 63.40 & YL32 \\
\hline 6.76 & 7.81 & 4.76 & 3.96 & 3.72 & 9.19 & 100.00 & 4.32 & 3.95 & 4.02 & 11.54 & 11.49 & YL44 \\
\hline 15.95 & 7.81 & 19.05 & 7.16 & 7.16 & 21.69 & 19.05 & 100.00 & 12.79 & 7.07 & 10.00 & 21.28 & YL45 \\
\hline 56.76 & 27.34 & 44.76 & 18.90 & 17.48 & 32.72 & 40.48 & 29.73 & 100.00 & 21.54 & 27.69 & 36.60 & I46 \\
\hline 26.76 & 59.38 & 64.76 & 66.31 & 49.86 & 45.22 & 59.52 & 23.78 & 31.16 & 100.00 & 45.38 & 48.94 & I48 \\
\hline 23.24 & 23.44 & 12.38 & 10.82 & 13.47 & 29.78 & 35.71 & 7.03 & 8.37 & 9.49 & 100.00 & 31.06 & I49 \\
\hline 34.32 & 36.72 & 20.95 & 18.90 & 14.90 & 54.78 & 64.29 & 27.03 & 20.00 & 18.49 & 56.15 & 100.00 & YL58 \\
\hline & & & & & & & & & & & & &
\end{tabular}

overlap of strain

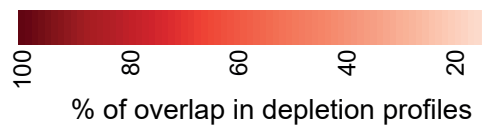

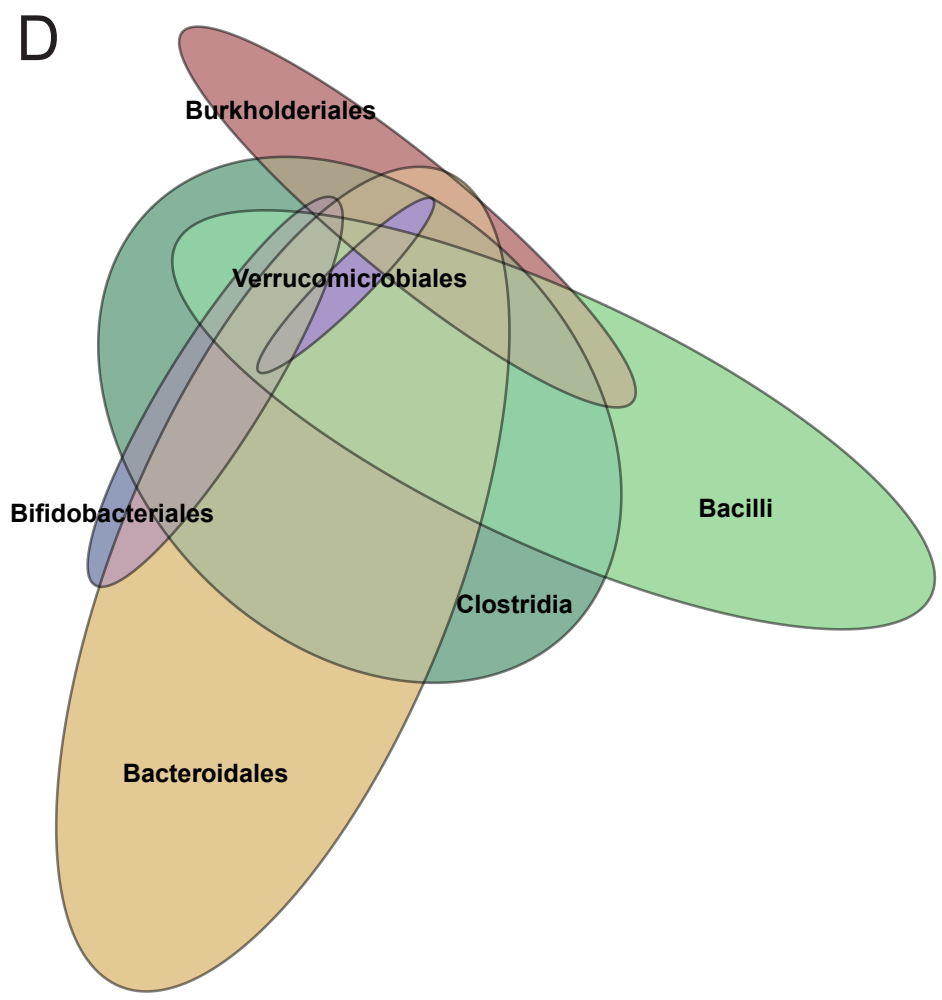


bioRxiv preprint doi: https://doi.org/10.1101/2021.02.25.432904; this version posted February 26, 2021. The copyright holder for this

preprint (which was not certified by peer review) is the author/funder. All rights reserved. No reuse allowed without permission.

\section{Figure 3}

A

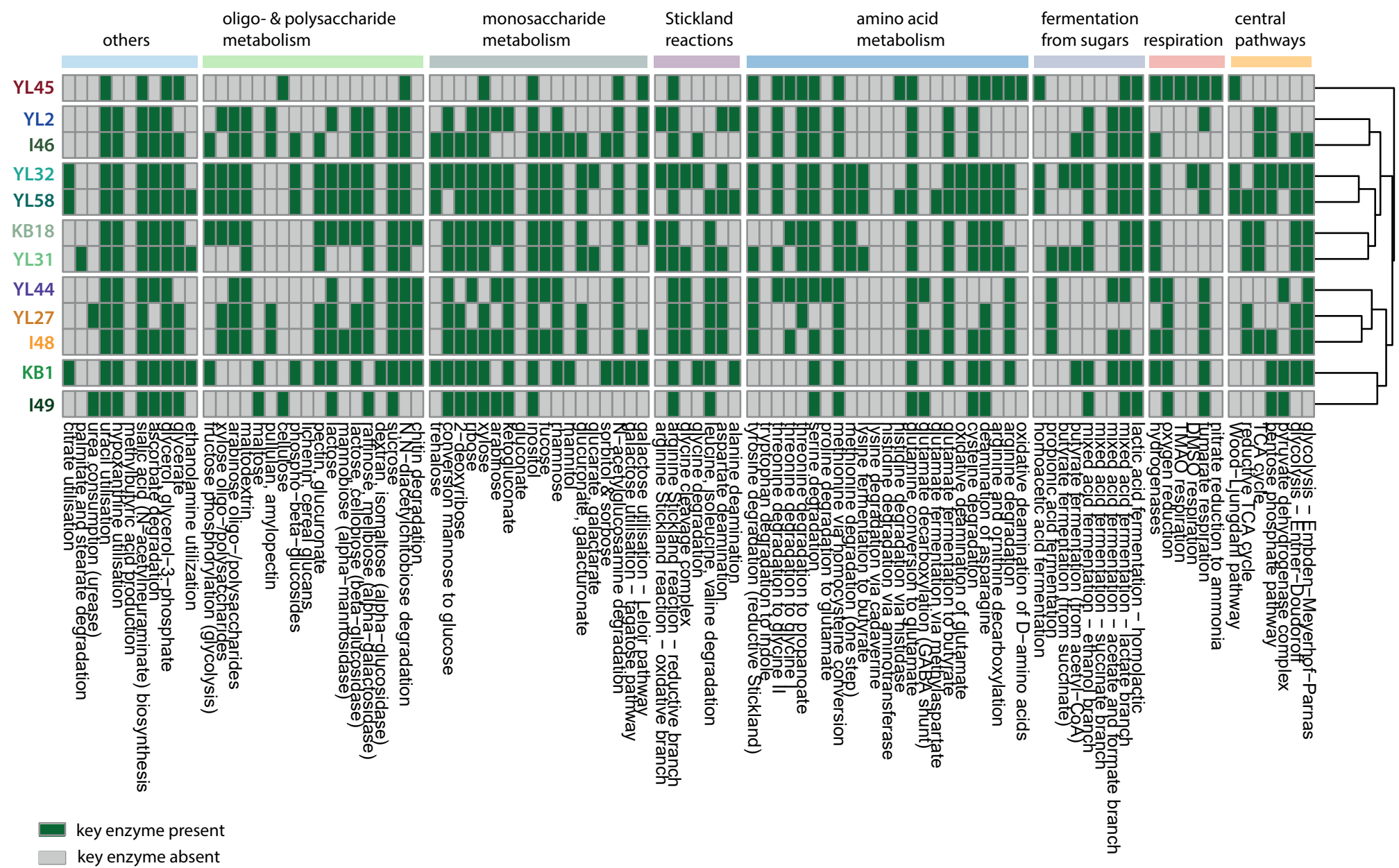

B

Enterococcus faecalis KB1

C
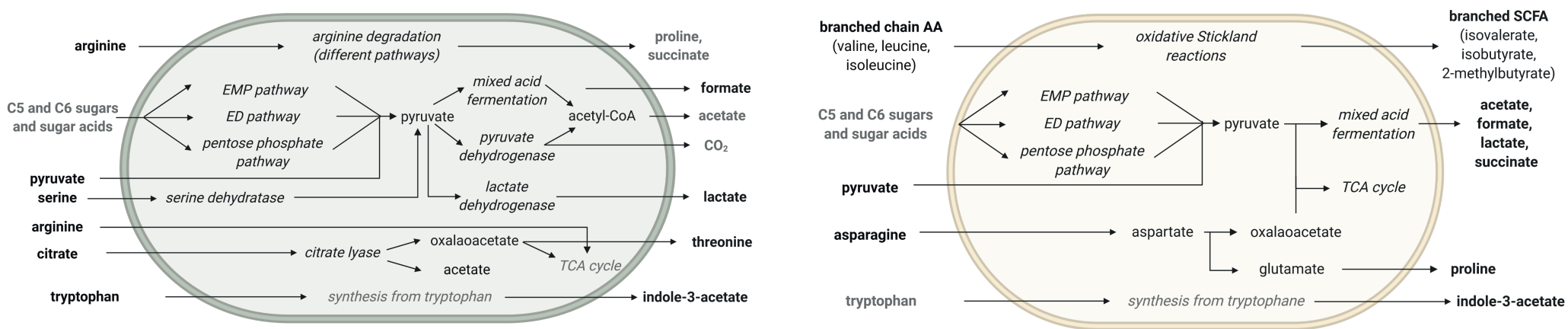

D

Blautia coccoides YL58

E

Flavonifractor plautii YL31
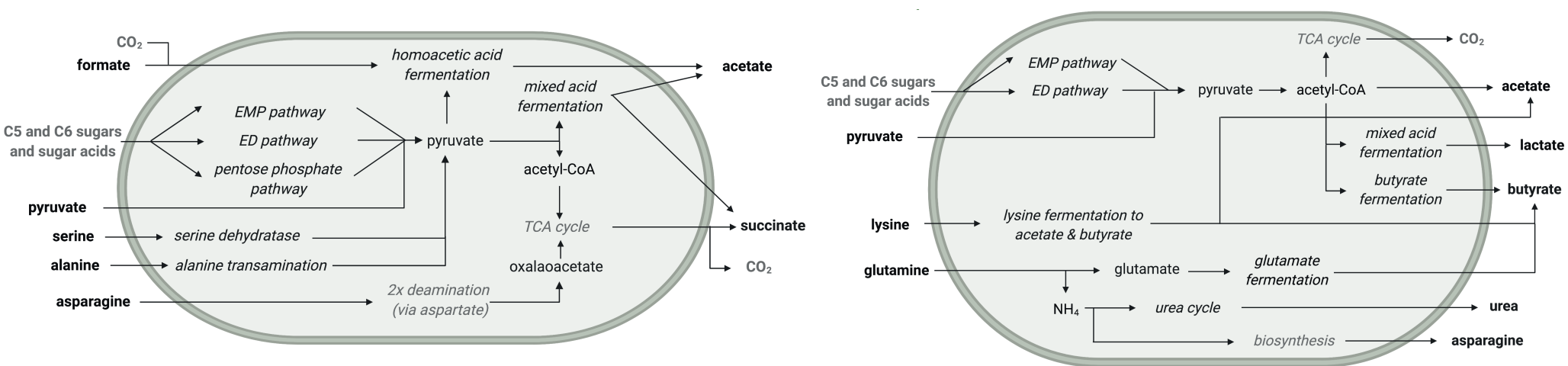
A

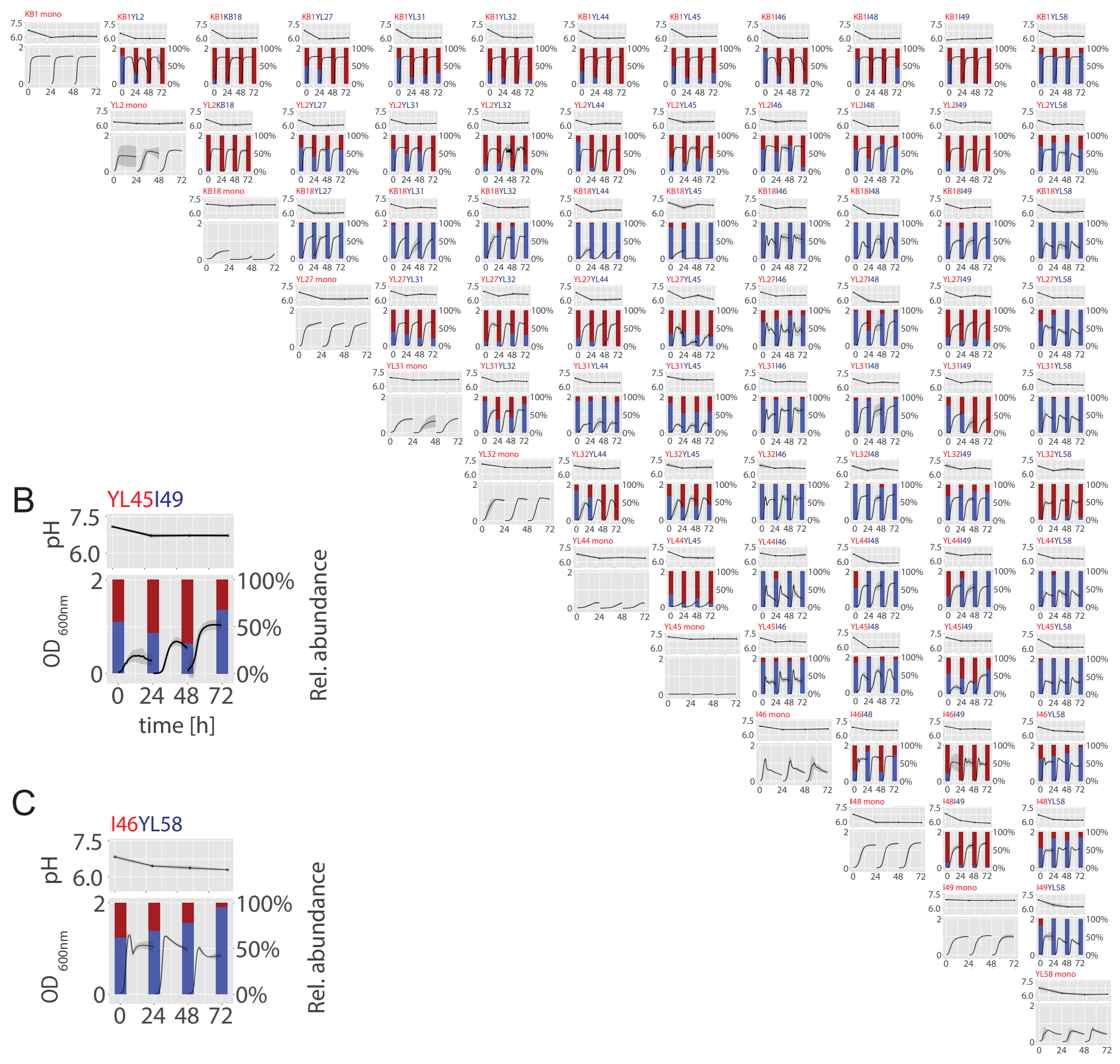


Figure 5

A

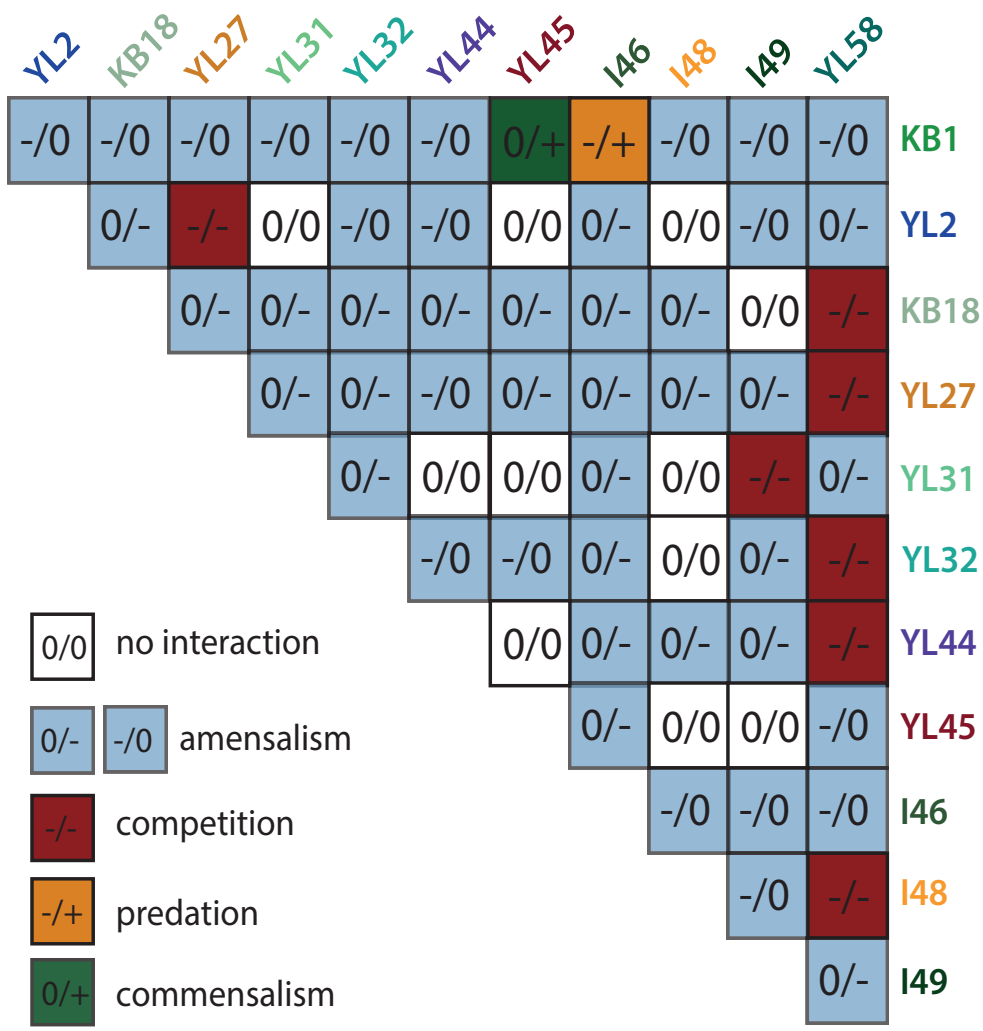

D Hypothesised interaction mechanism:

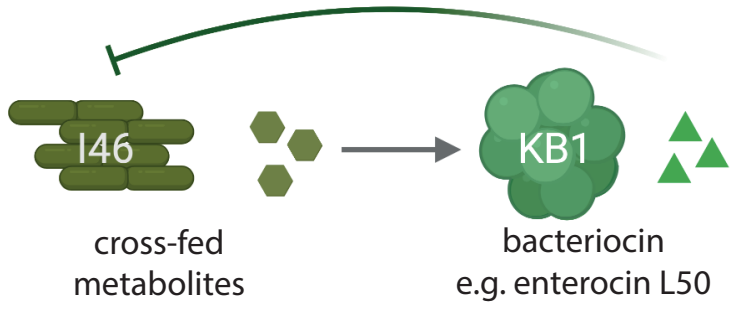

E

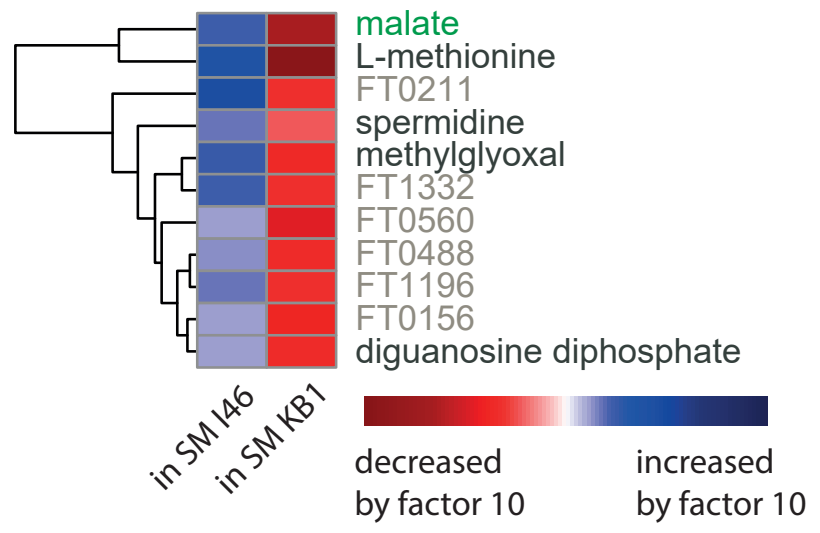

B

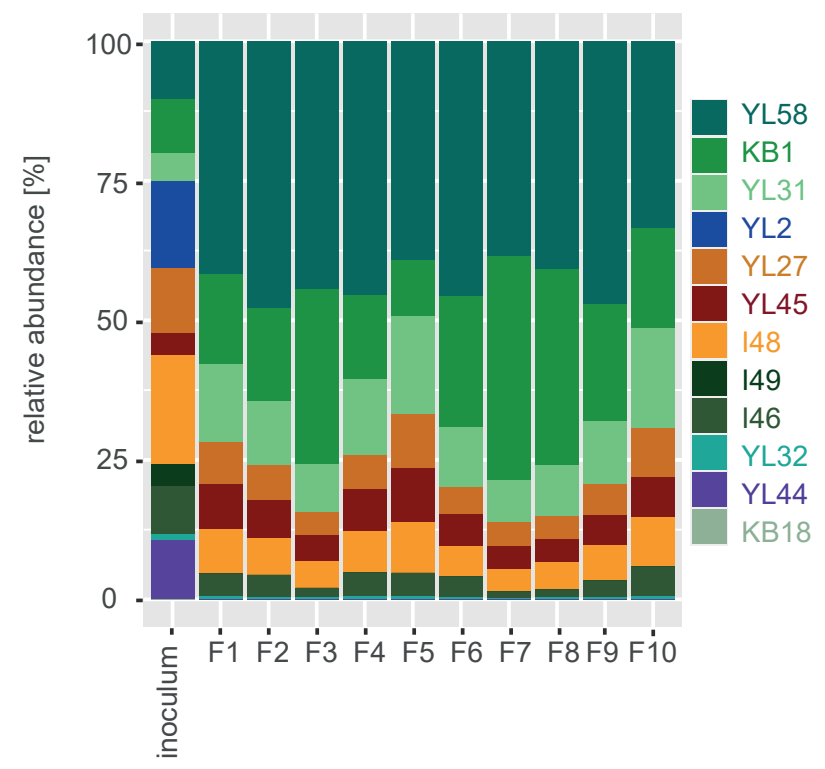

C

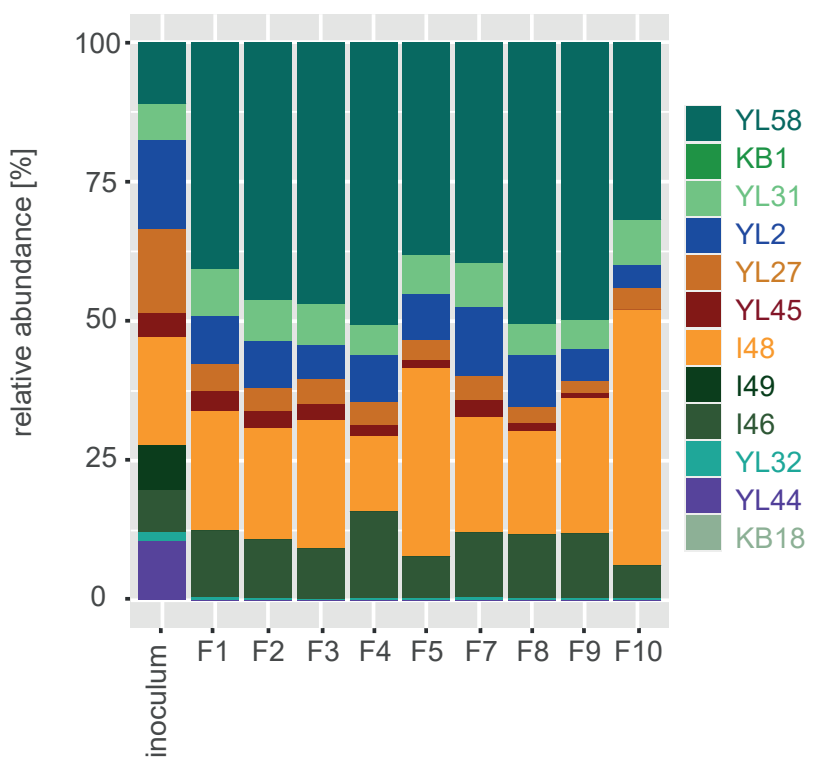

F

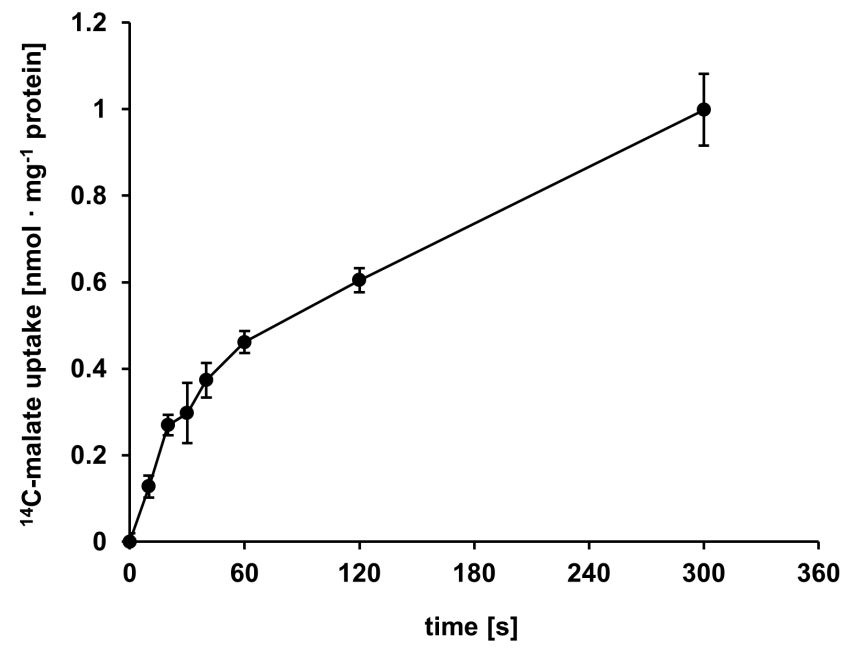

